\title{
Diatomeas perifíticas de un río de montaña (Tucumán, Argentina)
}

\section{Periphytic diatoms of a mountain river (Tucumán, Argentina)}

Nieva, Ángela M. ${ }^{1}$; Claudia T. Seeligmannn ${ }^{1 *}$; Nora I. Maidana ${ }^{2,3}$

1 Dpto. de Biología, Facultad de Cs. Naturales e Instituto Miguel Lillo. UNT. Miguel Lillo 205, (4000) S. M. de Tucumán, Argentina.

2 Universidad de Buenos Aires, Facultad de Ciencias Exactas y Naturales, Departamento de Biodiversidad y Biología Experimental, Laboratorio de Diatomeas Continentales, Buenos Aires, Argentina.

3 CONICET - Universidad de Buenos Aires, Instituto de Biodiversidad y Biología Experimental y Aplicada (IBBEA), Laboratorio de Diatomeas Continentales, Buenos Aires, Argentina.

* Autor corresponsal: claudiaseeligmann@gmail.com

\section{RESUMEN}

En el marco del estudio de la diatomoflora perifítica (epiliton y epifiton) del río San Javier (Tucumán, Argentina) identificamos 40 géneros y 115 taxones infragenéricos, de los cuales 5 son nuevos registros para Argentina: Gomphonema lippertii, Navicula densilineolata, Placomeis neoexigua, Stauroneis adamsiana y Ulnaria ramesi. Además, 6 son nuevas citas para Tucumán: Encyonema neomesianum, Halamphora normanii, Hantzschia abundans, Navicula antonii, N. rostellata y Placoneis symmetrica. Observamos que, en el periodo estudiado, el incremento de conductividad producida por el ingreso de agua desde un tributario provocó una disminución en la riqueza específica del epifiton y en la abundancia de ambas comunidades.

Palabras clave - Bacillariophycaeae, conductividad, río, salinidad.

\section{ABSTRACT}

In the framework of the study of the periphytic diatomoflora (epiliton and epiphyton) of the San Javier River (Tucumán, Argentina) we identified 40 genera and 115 infrageneric taxa of which 5 are new records for Argentina: Gomphonema lippertii, Navicula densilineolata, Placomeis neoexigua, Stauroneis adamsiana y Ulnaria ramesi. In addition, 6 are new records for Tucumán: Encyonema neomesianum, Halamphora

Ref. bibliográfica: Nieva, Á. M.; Seeligmann, C. T.; Maidana, N. I. 2019. "Diatomeas perifíticas de un río de montaña (Tucumán, Argentina)". Lilloa 56 (1): 64-91. Fundación Miguel Lillo, Tucumán, Argentina. D.0.I.: doi.org/10.30550/j.lil/2019.56.1/5

> Recibido: 16/11/18 - Aceptado: 08/04/19

- URL de la revista: http://lilloa.lillo.org.ar

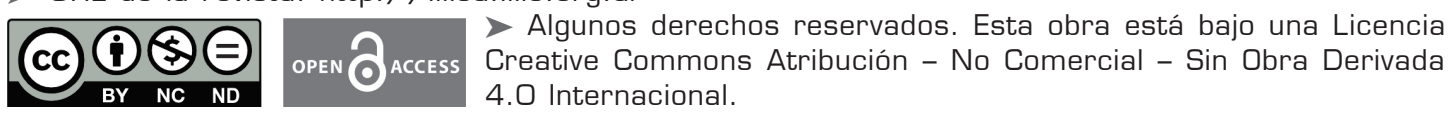


normanii, Hantzschia abundans, Navicula antonii, N. rostellata y Placoneis symmetrica. We observe that the increase in conductivity produced by the water input from a tributary caused a decrease in the specific richness of the epiphyton and in the abundance of both communities.

Keywords - Bacillariophycaeae, conductivity, river, salinity.

\section{INTRODUCCIÓN}

Entre las comunidades que podemos encontrar en ambientes dulceacuícolas, el perifiton cobra singular importancia ecológica por la elevada productividad primaria que aporta y por servir de alimento y refugio a numerosos organismos bentónicos. Wetzel (1983) define al perifiton como la compleja comunidad de microbiota (bacterias, hongos, algas, protozoos y animales) y detritos orgánicos e inorgánicos, adheridos a un sustrato sumergido, natural o artificial, vivo o muerto. Esta comunidad, también llamada biofilm (Burns y Ryder, 2001) cubre rocas, madera, partículas de sedimento, etc. La composición taxonómica del perifiton es utilizada para evaluar el estado trófico, el grado y tipo de contaminación de diversos ecosistemas acuáticos y la calidad de agua (Tell y Mazzoni, 1995, Licursi y Gómez, 2003, Pizarro y Alemanni, 2005). El desarrollo del conocimiento sobre la comunidad perifítica de un río contribuye, además, a entender las relaciones tróficas que son fundamentales para el funcionamiento del sistema.

Para estudiar la respuesta del perifiton a una perturbación, se pueden analizar sus aspectos estructurales o funcionales. Dentro de los primeros, además de la composición taxonómica y la diversidad específica, pueden evaluarse las distintas fracciones de su masa (autótrofa, heterótrofa e inorgánica) permitiendo inferir aspectos funcionales de la comunidad (Pizarro y Alemanni, 2005).

En los sistemas lóticos, las algas, y en especial las diatomeas, han sido ampliamente utilizadas como indicadores biológicos. Las diatomeas responden rápida y predeciblemente a los cambios ambientales como resultado de una serie compleja de interacciones entre la hidrología, la calidad de agua y los factores bióticos (Stevenson, Yangdong y Van Dam, 2010; Bellinger y Sigee, 2010, entre otros). Uno de los factores que probablemente influye más en las poblaciones de diatomeas es la salinidad. La mayoría de las especies son sensibles a las fluctuaciones de salinidad y es posible determinar el rango dentro del cual las poblaciones alcanzan su óptimo desarrollo (Stevenson, Peterson, Kirschtel, King, Tuchman, 1991).

Existen antecedentes de investigaciones previas realizadas en la cuenca del río Lules que permitieron ahondar en el conocimiento de la biodiversidad del bentos, su relación con el clima, las variaciones altitudinales, el impacto antrópico y el de las especies introducidas y definir grupos funcionales tróficos (Fernández y Barber, 2011). Sin embargo, hay un importante vacío de información respecto a la taxonomía y productividad algal perifítica en el río San Javier.

Los objetivos de este trabajo fueron dar a conocer la ficoflora diatomológica desarrollada sobre Cladophora sp. y sobre rocas en el río San Javier y detectar las va- 
riaciones en relación a la riqueza y abundancia de las dos comunidades que ocurren a lo largo de un gradiente de salinidad.

\section{MATERIALES Y MÉTODOS}

\section{Área de estudio}

El río San Javier es un curso de agua permanente de montaña que corre encajonado a espaldas de la Sierra de San Javier y recibe sobre su margen derecho (oeste) al arroyo Potrerillo a, $879 \mathrm{~m} \mathrm{snm}\left(26^{\circ} 47^{\prime} 04^{\prime} \mathrm{S}\right.$ y $\left.65^{\circ} 23^{\prime} 43^{\prime \prime} \mathrm{O}\right)$. Este arroyo tiene una elevada conductividad $\left(1200 \mu \mathrm{s} \mathrm{cm}^{-1}\right)$ aportada por sales de origen natural provenientes de sedimentos de la Formación Río Salí que está constituida, en parte, por calizas y concreciones yesíferas (Georgeff, Ibañez, Vides, Anis, Nieva, 2014). El área de estudio abarca al río San Javier en sus tramos anterior y posterior a la entrada del arroyo (Fig. 1A, 1B).

El clima es subtropical con un régimen monzónico de precipitaciones (inviernos secos, veranos lluviosos). La temperatura y las precipitaciones son fuertemente controladas por la topografía. La temperatura es máxima en el pedemonte oriental (19 ${ }^{\circ} \mathrm{C}$ de temperatura media anual) y mínima en las zonas más altas (estimativamente, $12{ }^{\circ} \mathrm{C}$ ). Las precipitaciones probablemente alcanzan su máximo valor alrededor de los $1000 \mathrm{~m}$ en las laderas orientales de la sierra (estimativamente, más de $1500 \mathrm{~mm}$ anuales) y su mínimo valor en el norte (alrededor de $500 \mathrm{~mm}$ anuales). Durante la segunda mitad del siglo XX, las precipitaciones se incrementaron regionalmente cerca de un 20 \% (Minetti y Vargas, 1997). El grueso de la vegetación natural corresponde a la provincia fitogeográfica de las Yungas, con sectores correspondientes al Chaco serrano en el sector norte de la sierra. Las Yungas se desarrollan sobre la vertiente oriental de las cadenas montañosas de los Andes desde Colombia y Venezuela hasta la Argentina. También pueden ser llamadas globalmente como Bosques Andinos Yungueños (Malizia et al., 2005) definidos principalmente por ocurrir en las laderas de las montañas en una franja altitudinal caracterizada por una persistente o estacional cobertura de nubes y neblinas. Los bosques nublados tienen una enorme diversidad biológica y son reguladores de importantes caudales hídricos que atraviesan el continente americano. En la actualidad, los bosques nublados están considerados como uno de los sistemas naturales más frágiles a la intervención humana especialmente por procesos de degradación, sobreutilización y conversión en sistemas agrícolas y de pastoreo (Brown, Pacheco, Lomáscolo, Malizia, 2006)

El muestreo para el relevamiento de las diatomeas se realizó en diciembre de 2014, en el tramo del río San Javier donde desemboca el arroyo Potrerillo. Se seleccionaron cinco sitios a lo largo de $100 \mathrm{~m}$, teniendo en cuenta el gradiente de conductividad previamente determinado (Fig. 1, Tabla 1): antes de la confluencia con el arroyo Potrerillo (sitio 1) y después del ingreso del arroyo (sitios 2 a 5). Se evaluaron in situ el ancho del cauce, la profundidad y la velocidad de la corriente (con el método del flotante). Además, se midieron la temperatura, la conductividad y el oxígeno disuelto con analizador de agua multiparamétrico, digital, portátil, marca 


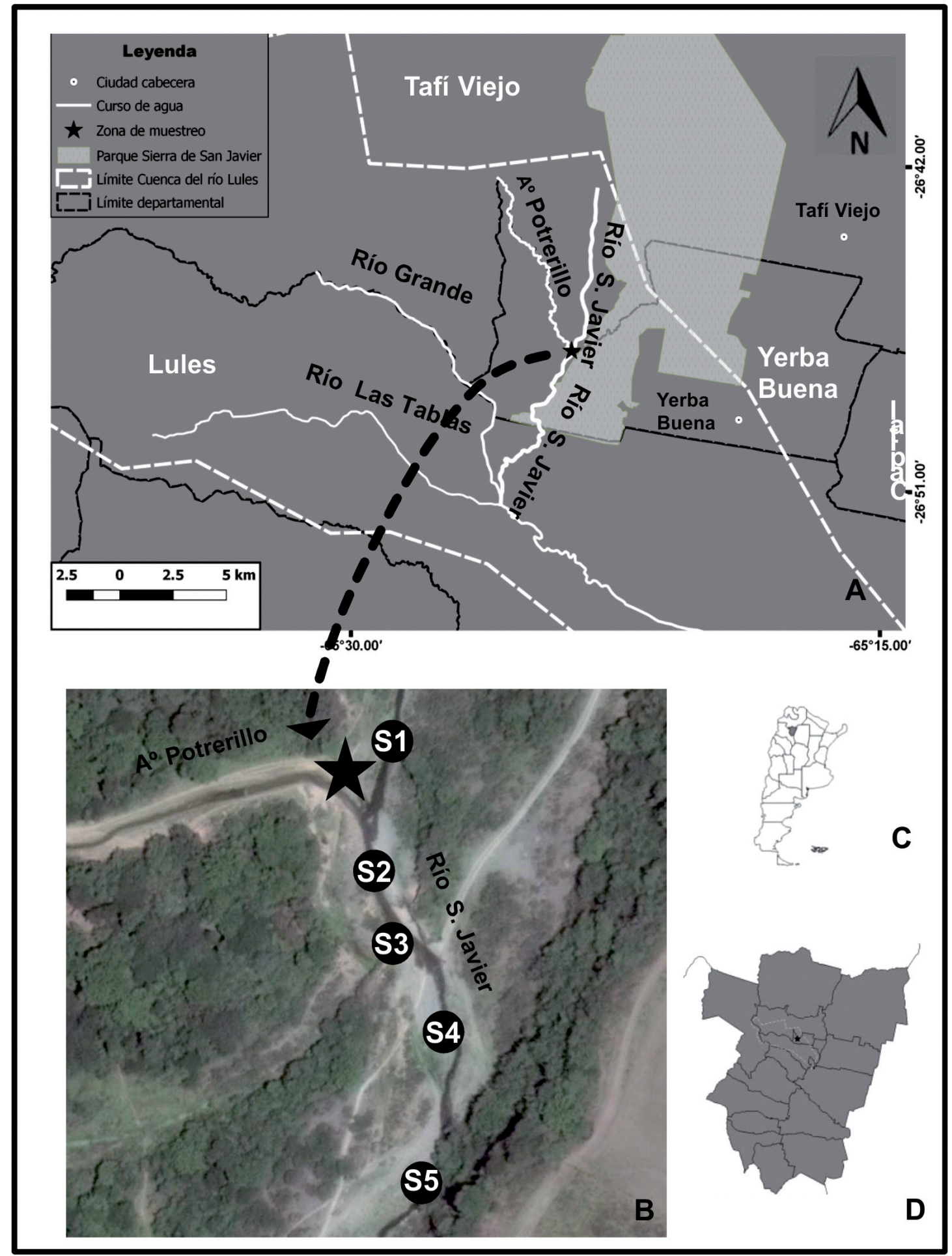

Fig. 1. Mapa de ubicación de los sitios de muestreo. A) Área de estudio. B) Detalle de la zona de estudio y ubicación de sitios de muestro: (1): antes de la entrada del arroyo Potrerillo, (2-5): posterior a la entrada del arroyo Potrerillo. C: mapa de Argentina. D: mapa de la provincia de Tucumán. 
Tabla 1. Variables fisicoquímicas medidas en el río San Javier para diciembre de 2014. Prof. prom. = profundidad promedio; Vel. prom. = velocidad promedio; $\mathrm{T}=$ temperatura; Cond. $=$ conductividad; OD = oxígeno disuelto.

\begin{tabular}{|c|c|c|c|c|c|c|c|c|c|}
\hline Sitios & $\begin{array}{c}\text { Ancho } \\
\text { cauce } \\
(\mathrm{m})\end{array}$ & $\begin{array}{l}\text { Prof. prom. } \\
\quad(\mathrm{cm})\end{array}$ & $\begin{array}{l}\text { Vel. prom. } \\
\left(\mathrm{cm} \mathrm{s}^{-1}\right)\end{array}$ & Coordenadas & Zona & $\begin{array}{c}\mathrm{T} \\
\left({ }^{\circ} \mathrm{C}\right)\end{array}$ & $\mathrm{pH}$ & $\begin{array}{l}\text { Cond. } \\
\left(\mu \mathrm{sm}^{-1}\right)\end{array}$ & $\begin{array}{c}\mathrm{OD} \\
\left(\mathrm{mg} \mathrm{l}^{-1}\right)\end{array}$ \\
\hline 1 & 3,3 & 12,4 & 7,4 & $\begin{array}{c}26^{\circ} 47^{\circ} 04.1^{\prime \prime} \\
\mathrm{S} \\
65^{\circ} 23^{\prime} 44.2^{\prime \prime} \\
\mathrm{O}\end{array}$ & $\begin{array}{c}\text { MI } \\
\mathrm{C} \\
\mathrm{MD}\end{array}$ & $\begin{array}{l}23,2 \\
23,2 \\
23,2\end{array}$ & $\begin{array}{l}7 \\
7 \\
7\end{array}$ & $\begin{array}{l}309 \\
307 \\
309\end{array}$ & $\begin{array}{c}12,3 \\
12\end{array}$ \\
\hline 2 & 3,7 & 11,1 & 7 & $\begin{array}{c}26^{\circ} 47^{\prime} 05,6^{\prime \prime} \\
S \\
65^{\circ} 23^{\prime} 44,5^{\prime \prime} \\
O\end{array}$ & $\begin{array}{c}\text { MI } \\
\mathrm{C} \\
\mathrm{MD}\end{array}$ & $\begin{array}{l}22,6 \\
22,3 \\
22,6\end{array}$ & $\begin{array}{l}7 \\
8 \\
8\end{array}$ & $\begin{array}{c}309 \\
471 \\
1146\end{array}$ & $\begin{array}{l}12,5 \\
11,5 \\
11,2\end{array}$ \\
\hline 3 & 5,4 & 12,2 & 9,7 & $\begin{array}{c}26^{\circ} 47^{\prime} 06,5^{\prime \prime} \mathrm{S} \\
-65^{\circ} 23^{\prime} 44,2^{\prime \prime} \\
\mathrm{O}\end{array}$ & $\begin{array}{c}\text { MI } \\
\text { C } \\
\text { MD }\end{array}$ & $\begin{array}{l}22,1 \\
22,3 \\
22,4\end{array}$ & $\begin{array}{l}8 \\
7 \\
8\end{array}$ & $\begin{array}{l}376 \\
749 \\
896 \\
\end{array}$ & $\begin{array}{l}12,2 \\
11,7 \\
11,3\end{array}$ \\
\hline 4 & 5,9 & 9,6 & 8 & $\begin{array}{c}26^{\circ} 47^{\circ} 07,7^{\prime \prime} \\
S \\
65^{\circ} 23^{\circ} 43,5^{\prime \prime} \\
O\end{array}$ & $\begin{array}{c}\mathrm{MI} \\
\mathrm{C} \\
\mathrm{MD}\end{array}$ & $\begin{array}{l}22,1 \\
22,1 \\
22,1\end{array}$ & $\begin{array}{l}7 \\
7 \\
8\end{array}$ & $\begin{array}{l}553 \\
754 \\
801\end{array}$ & $\begin{array}{c}11 \\
11 \\
10,4\end{array}$ \\
\hline 5 & 2,6 & 27,4 & 6,7 & $\begin{array}{c}26^{\circ} 47^{\prime} 08,1^{\prime \prime} \\
S \\
65^{\circ} 23^{\circ} 43,5^{\prime \prime} \\
O\end{array}$ & $\begin{array}{c}\text { MI } \\
\mathrm{C} \\
\mathrm{MD}\end{array}$ & $\begin{array}{l}21,9 \\
21,9 \\
21,9\end{array}$ & $\begin{array}{l}7 \\
7 \\
8\end{array}$ & $\begin{array}{l}516 \\
549 \\
727\end{array}$ & $\begin{array}{l}11,2 \\
10,5 \\
10,2\end{array}$ \\
\hline
\end{tabular}

Metre 850081 SperScientific (Tabla 1). En todos los sitios, se seleccionaron 3 rocas de los márgenes y del centro del cauce del río para los análisis cuali y cuantitativos del epiliton. En cada roca se delimitó, con una plantilla plástica, una superficie de $10 \mathrm{~cm}^{2}$, que se raspó utilizando un cepillo de cerdas. De esta manera, se obtuvo de cada sitio una muestra integrada de $30 \mathrm{~cm}^{2}$ de superficie raspada que se fijó con formol al $4 \%$ y se colocó en un envase cónico de $15 \mathrm{ml}$ con $10 \mathrm{ml}$ de agua destilada colocada previamente.

El alga verde Cladophora Kútzing fue el sustrato seleccionado para el estudio del epifiton por ser muy frecuente en el río. En cada sitio se recogieron 3 rocas sumergidas ( 3 en cada margen y 3 en el centro del cauce del río), que tuvieran talos adheridos y que estuvieran ubicadas de tal manera que pudiera trazarse una transecta transversal respecto a la dirección de la corriente. Se extrajeron porciones de talos para obtener en total 3 muestras integradas ( 1 para cada margen y 1 para el centro del cauce del río) las que fueron lavadas repetidas veces para obtener solo algas epifíticas. Las muestras se colocaron en tubos cónicos de $15 \mathrm{ml}$ (con $10 \mathrm{ml}$ de agua destilada) y se fijaron con formol al $4 \%$. En el laboratorio, los talos fueron deshidratados en una estufa a $60^{\circ} \mathrm{C}$ durante 24 horas. Posteriormente, se registró el peso seco de cada muestra. A continuación se destruyeron los talos del alga verde y otros epífitos no silíceos mediante una digestión con ácido sulfúrico al 98 \% con la finalidad de obtener solamente los frústulos de las diatomeas epífitas. Luego de sucesivos lavados con agua destilada, el material se colocó en envases con $10 \mathrm{ml}$ de agua destilada para el posterior análisis cuali y cuantitativo.

Con el material tratado se hicieron preparaciones permanentes utilizando Naphrax ${ }^{\circledR}$ como medio de montaje. Las observaciones se realizaron bajo microscopio 
óptico binocular Leica modelo DM LS 2, provisto de una cámara digital Canon PowerShot S50. El análisis cuantitativo de la diatomoflora sobre Cladophora sp. y sobre rocas (número de valvas/g de peso seco y número de valvas $/ \mathrm{cm}^{2}$ de roca, respectivamente) se realizó a partir de la aplicación del método de las transectas descripto en Villafañe y Reid (1995) con modificaciones. La identificación taxonómica se basó, en general, en las monografías de Hartley (1996), Lange-Bertalot, 1999, Metzeltin y Lange-Bertalot (1998, 2007), Metzeltin, Lange-Bertalot y García-Rodríguez (2005), Patrick (1961), Patrick y Reimer (1966, 1975), Rumrich, Lange-Bertalot y Rumrich (2000), Krammer y Lange-Bertalot $(1986,1988,1991,2000,2004)$ y obras específicas de autores varios que se mencionan en cada caso. Para la distribución geográfica en Argentina se consultó a Luchini y Verona (1972), Vouilloud (2003) y publicaciones específicas de autores varios.

Los requerimientos ecológicos de las distintas especies fueron tomados de De Wolf (1982), Van Dam, Mertens, Sinkeldam (1994), Lowe (1974) y de la literatura consultada en cada caso.

Las muestras recolectadas fueron incorporadas a la Colección Ficológica (LIL) de la Fundación Miguel Lillo.

Se utilizó el software de hidroquímica Diagrammes (Simler, 2009) para obtener el diagrama de Stiff.

\section{RESULTADOS}

A partir del punto de encuentro del río San Javier con el arroyo Potrerillo (sitio 2), se registraron cambios importantes en los valores de conductividad (de 300

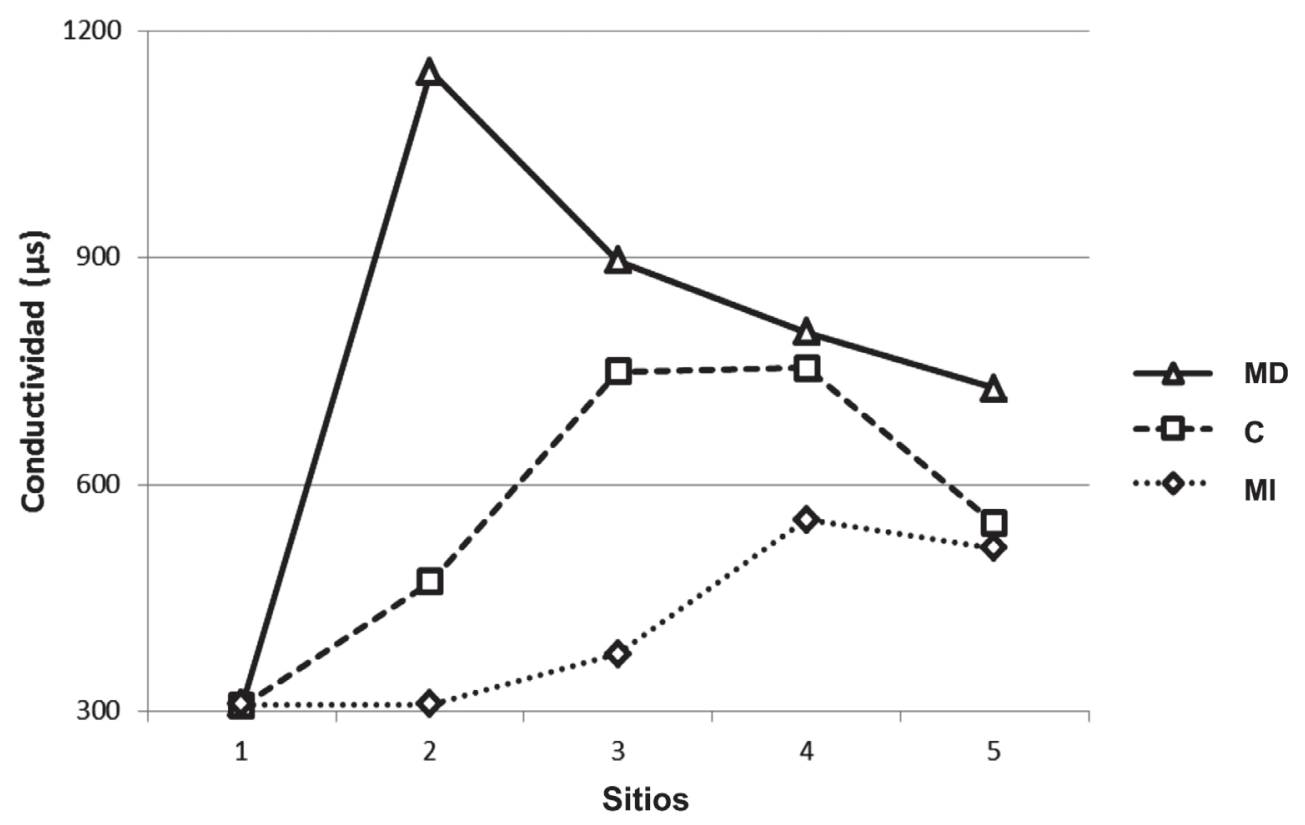

Fig. 2. Conductividad medida en los cinco sitios de muestreo en el margen derecho (MD), margen izquierdo (MI) y en el centro (C) del cauce del río San Javier. 
$\mu \mathrm{sm}^{-1}$ a $1146 \mu \mathrm{s} \mathrm{cm}^{-1}$ ), especialmente debido al aumento de los sulfatos (Tabla 1). El incremento de la conductividad fue mayor en el margen derecho (MD), por la ubicación del ingreso del arroyo y disminuyó desde el centro (C) hacia el margen izquierdo (MI) (Fig. 2). También se detectaron, con el ingreso del arroyo, en el sitio 3 , incrementos en la velocidad de la corriente (Tabla 1).

El diagrama de Stiff (Fig. 3) realizado para los sitios 1, 2 y 4 muestra que las aguas del río San Javier son bicarbonatadas-sulfatadas-cálcicas-sódicas antes del encuentro con el arroyo Potrerillo (sitiol) y, posteriormente, se tornan sulfatadasbicarbonatadas-cálcicas-sódicas (a partir del sitio 2) acompañado por un incremento del pH (Tabla 1).

Dentro de las diatomeas epilíticas y epifíticas se identificaron 40 géneros y 115 taxones infragenéricos. Los géneros mejor representados en cuanto al número de especies fueron Nitzschia (21), Gomphonema (12), Navicula (8), Luticola (6), Surirella (5) y Halamphora (4). De las 115 especies encontradas en la transecta analizada, 87 fueron comunes a ambas comunidades, 19 fueron halladas solo en el epiliton y 9 observadas solo en el epifiton (Tabla 2; Fig. 4).

Se observaron diferencias entre los sitios analizados, tanto en la comunidad epilítica como en la epifítica. Para el epiliton se encontró una riqueza específica menor en sitio 1, tanto en el centro como en el margen derecho respecto a los sitios ubicados aguas abajo (2-5) y en el margen izquierdo se hallaron 21 especies epilíticas menos que en el derecho (Fig. 5A). Para el epifiton se observó (tanto en los márgenes como en el centro) que el número de especies fue mayor antes de la entrada del arroyo (sitio 1) y no logró recuperarse a lo largo de la transecta (Fig. 5B). La abundancia de diatomeas disminuyó con el ingreso del arroyo en un $98 \%$ y un $89 \%$ para el epiliton y epifiton, respectivamente, sobre todo en el margen derecho

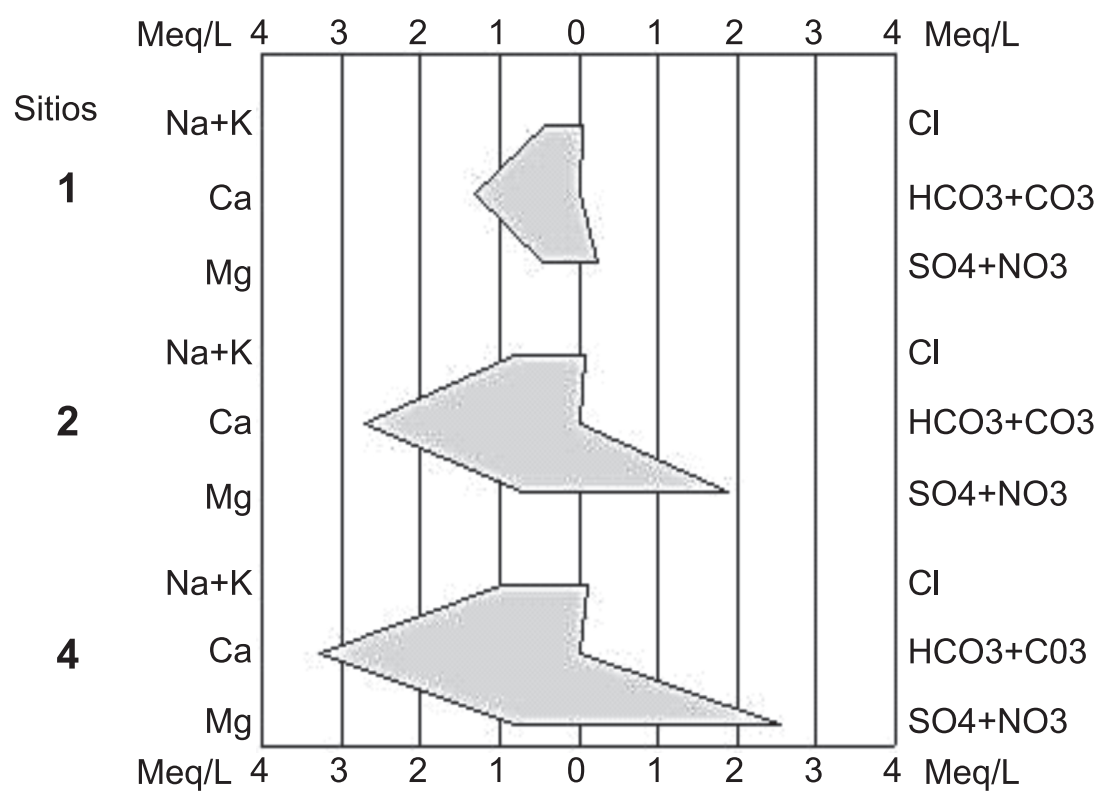

Fig. 3. Diagrama de Stiff para los sitios 1 (río arriba, antes de la unión con el arroyo), 2 (unión río y arroyo) y 4 (río abajo posterior a la unión con el arroyo) del río San Javier. 


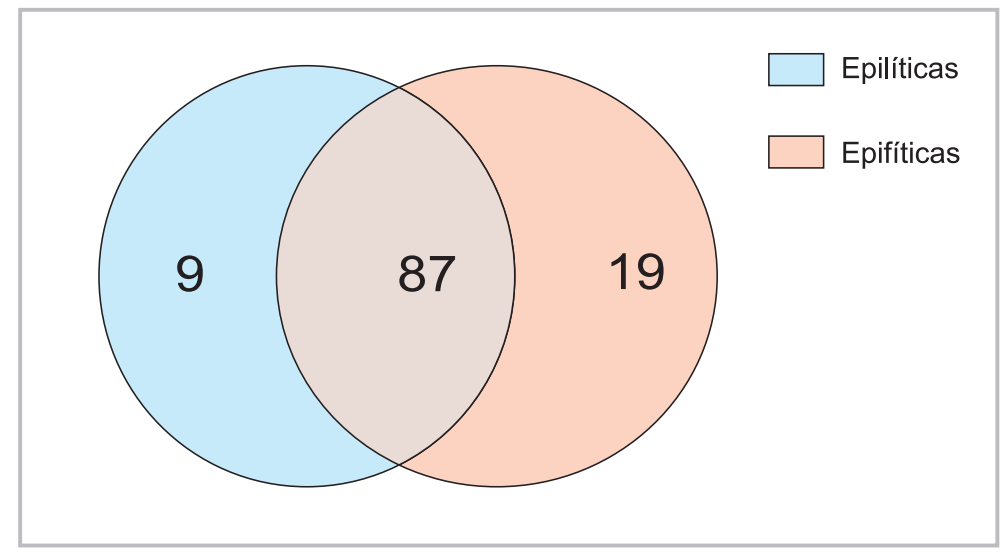

Fig. 4. Número total de especies epilíticas y epifíticas exclusivas y compartidas.
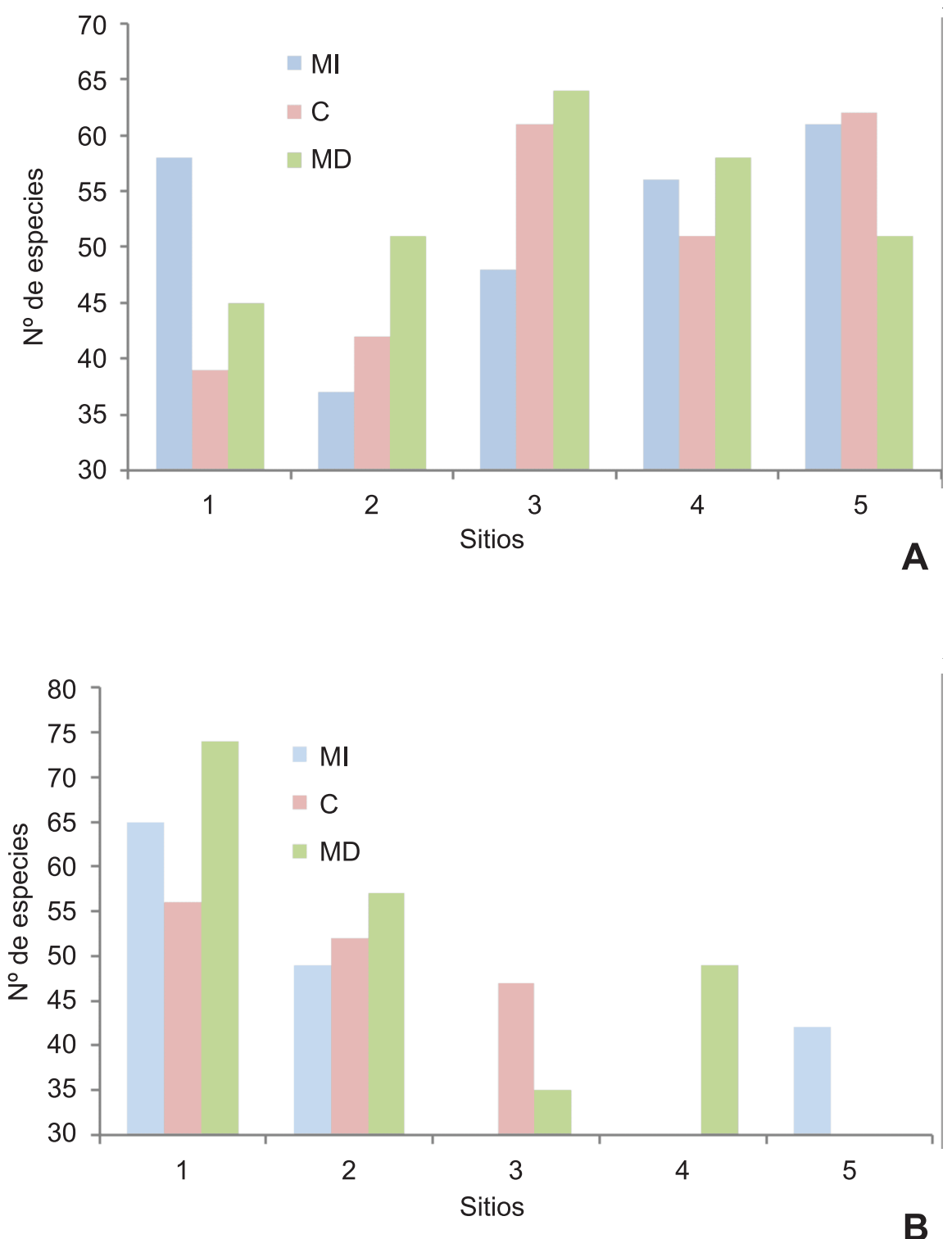

Fig. 5. Variación del número de especies en las dos comunidades. A) Epiliton. B) Epifiton. MI (margen izquierdo), C (centro) y MD (margen derecho). 
Tabla 2. Ocurrencia de los taxones epilíticos (*) y epifíticos ( $\bullet$ en el río San Javier. En el margen izquierdo (MI), centro (C) y margen derecho (MD). •• Nuevas citas para Argentina. • Nuevas citas para Tucumán.

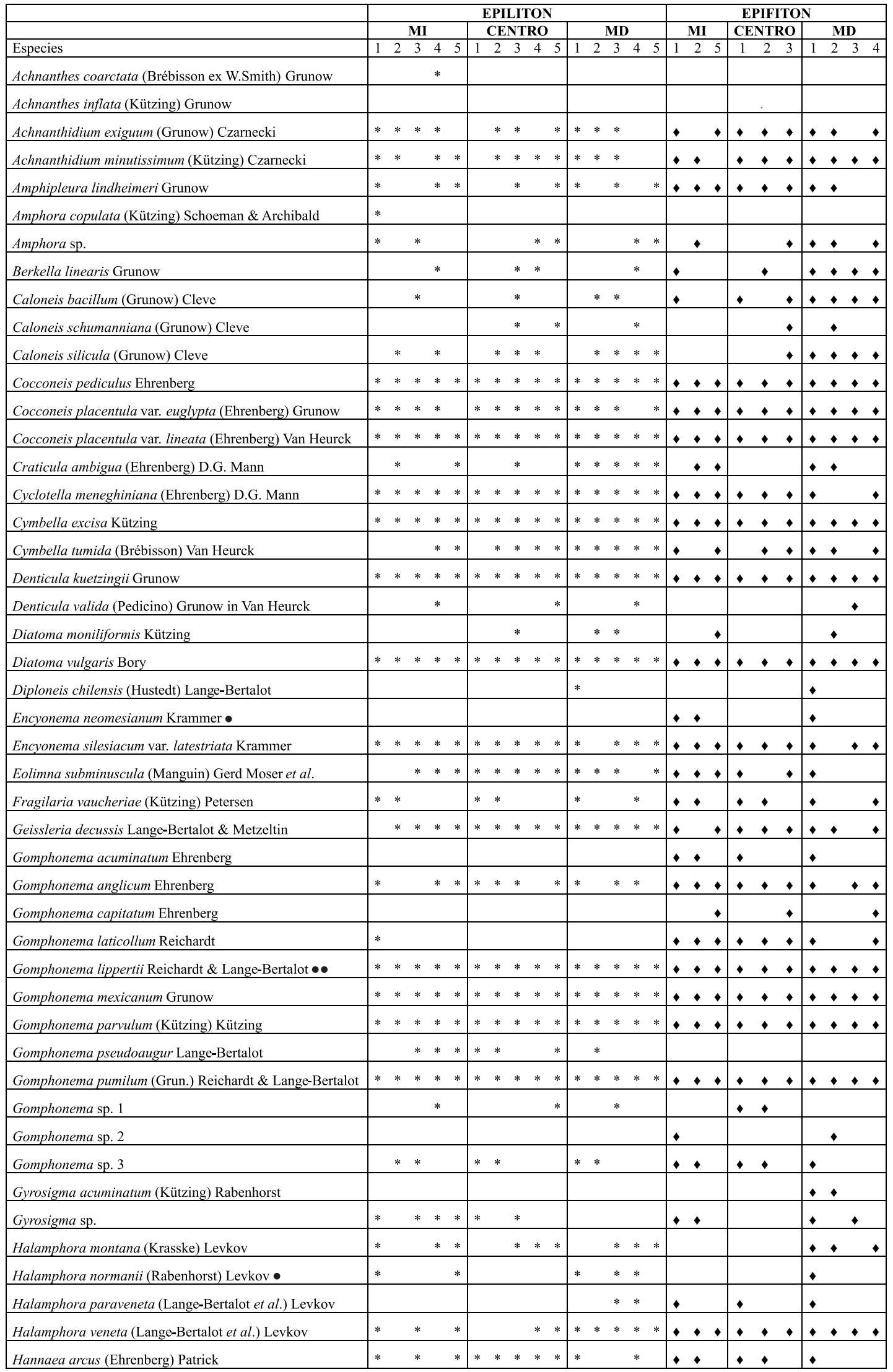


Tabla 2 (cont.).

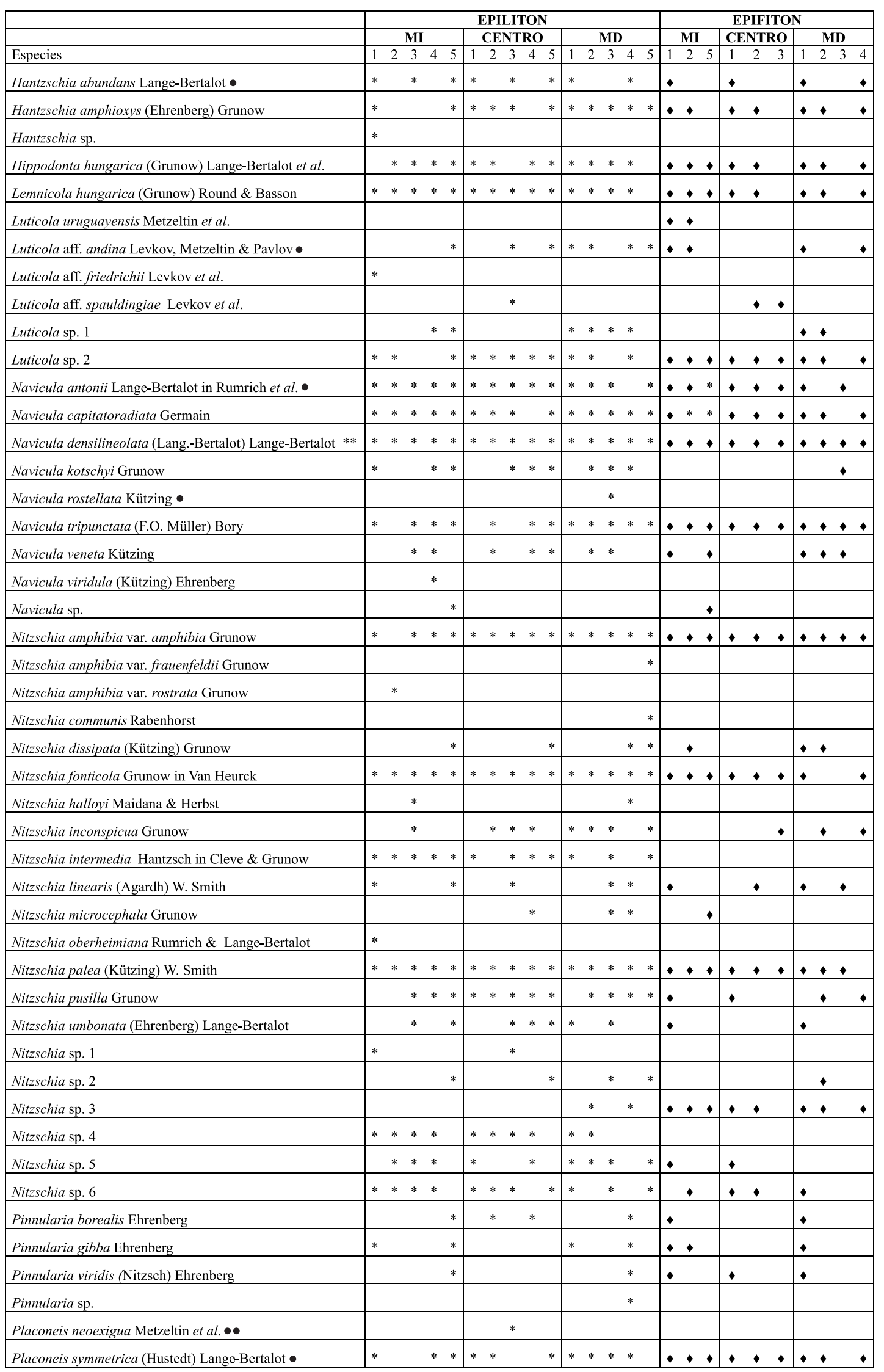


Tabla 2 (cont.).

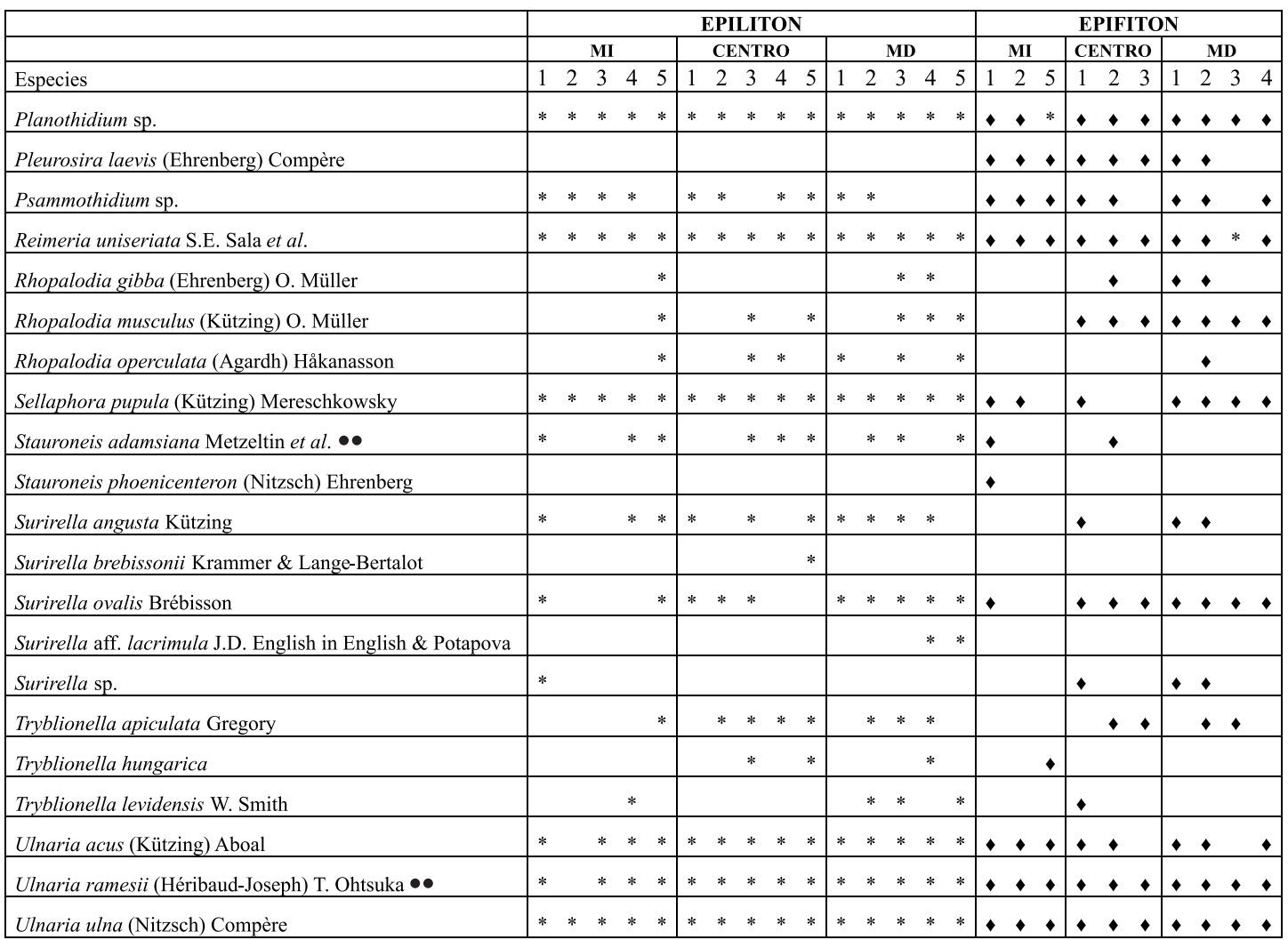

del río (Figs. 6A y 6B). Para el epiliton se hallaron con una abundancia relativa mayor al $4 \%$ en el sitio 1 a Cymbella excisa Kützing, Denticula kuetzingii Grunow, Gomphonema paroulum (Kützing) Kützing, Nitzschia amphibia Grunow var. amphibia, Nitzschia fonticola Grunow in Van Heurck y Nitzscha sp. 3 (Tabla 3). De estas especies continuaron, con un alto porcentaje en el sitio 2, C. excisa y G. paroulum. En el caso del epifiton, se destacaron por su abundancia relativa Achnanthidium minutissimum (Kützing) Czarnecki, Cocconeis pediculus Ehrenberg, Cymbella excisa, Gomphonema paroulum (Kützing) Kützing y Navicula antonii Lange-Bertalot en Rumrich et al., 2000 (Tabla 4).

A continuación se describen e ilustran algunas especies nuevas o poco conocidas para la provincia de Tucumán y el país.

\section{Encyonema neomesianum}

Krammer, 1997 (Figs. 7A, 7B)

Descripción.- Valvas semi-elípticas, dorsiventrales, con el lado dorsal fuertemente convexo y el ventral casi recto, con un ensanchamiento en la zona media. Extremos angostos, no rostrados. Ápices angostamente redondeados. Área axial angosta, lineal a lanceolada, desplazada hacia el lado ventral; área central ausente o pequeña. Rafe filiforme, con las fisuras proximales flexionadas hacia el lado dorsal. En general, 
Tabla 3. Abundancias relativas promedios de las especies mejor representadas en el epiliton.

\begin{tabular}{|l|c|c|c|c|c|}
\hline & Sitio 1 & Sitio 2 & Sitio 3 & Sitio 4 & Sitio 5 \\
\hline Cymbella excisa & 12,8 & 16,6 & 26,6 & 21,4 & 15,5 \\
Denticula kuetzingii & 4,2 & 1,92 & 1,93 & 1,2 & 9 \\
Gomphonema parvulum & 15 & 27,7 & 13,7 & 23,7 & 24,9 \\
Nitzschia amphibia & 25 & 5,4 & 3,1 & 2,9 & 2,55 \\
Nitzschia fonticola & 9,5 & 1,74 & 0,7 & 1,52 & 1,7 \\
Nitzschia sp.3 & 8,5 & 0,9 & 0,4 & 1,73 & 3,28 \\
\hline
\end{tabular}

Tabla 4. Abundancias relativas promedios de las especies mejor representadas en el epifiton.

\begin{tabular}{|l|c|c|c|c|c|}
\hline & Sitio 1 & Sitio 2 & Sitio 3 & Sitio 4 & Sitio 5 \\
\hline Ahnanthidium minutissimum & 1,94 & 8,2 & 11 & 14,2 & 1,4 \\
Cocconeis pediculus & 41,5 & 41,3 & 62 & 33,4 & 72,5 \\
Cymbella excisa & 5,38 & 12,3 & 8,3 & 14 & 6,6 \\
Gomphonema parvulum & 12,6 & 9,3 & 6,3 & 6,3 & 6,5 \\
Navicula antonii & 49 & 3 & 1,8 & 3 & 1 \\
\hline
\end{tabular}

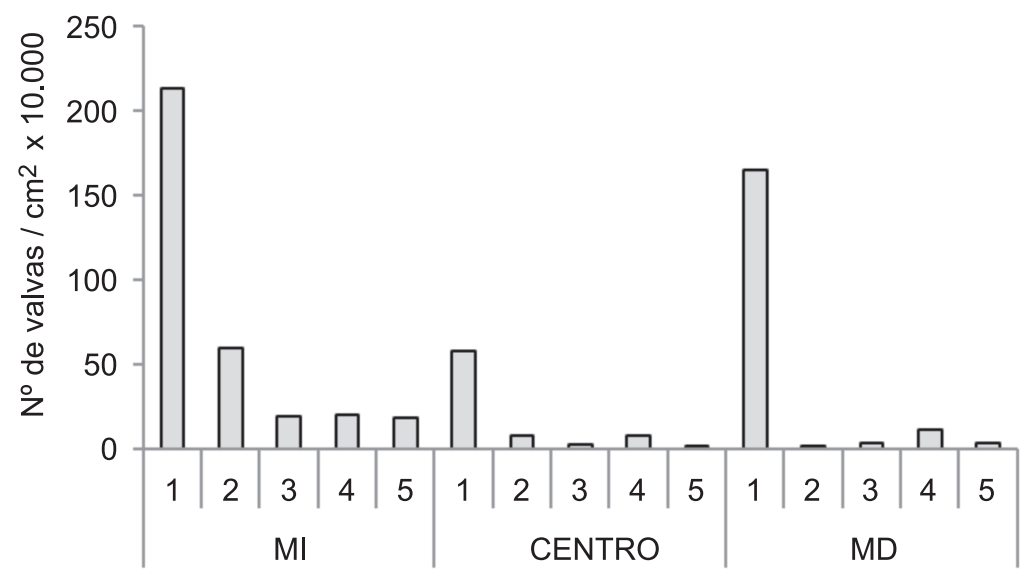

A

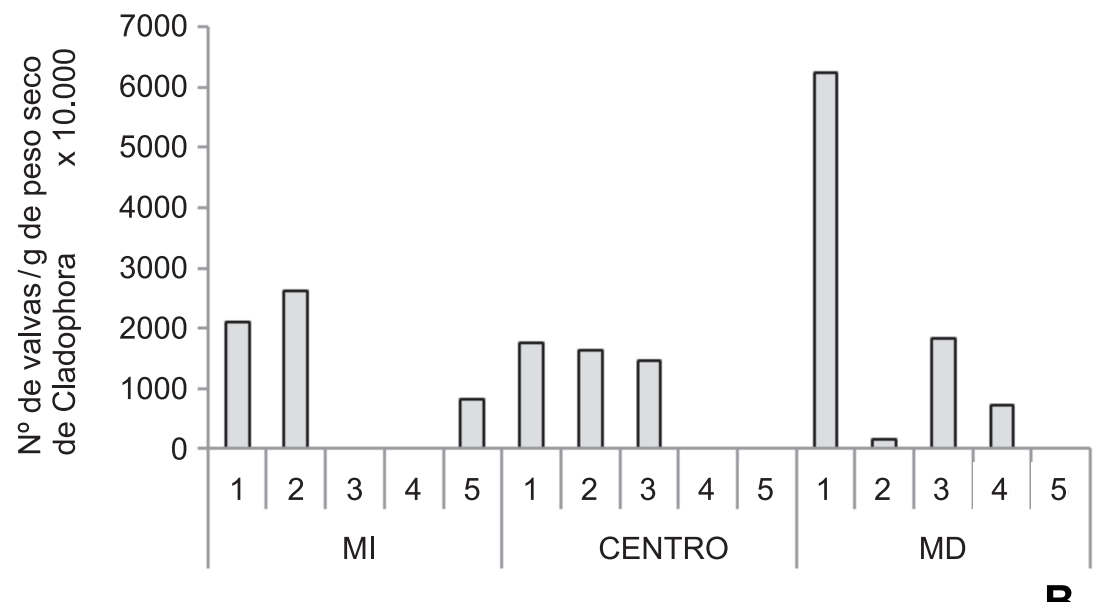

Fig. 6. Abundancia total de diatomeas en los sitios de muestreo del río San Javier. A) Del epiliton (número de valvas $/ \mathrm{cm}^{2}$ de roca). B) Del epifiton (número de valvas $/ \mathrm{g}$ de peso seco de Cladophora sp.). 
sin estigmoides dorsales. Estrías ligeramente radiales a radiales en la zona media de la valva, convergentes cerca de los ápices, finamente aereoladas.

Dimensiones. - Eje apical (EA) 43,8-60,9 $\mu \mathrm{m}$; eje transapical (ET) 10,9-12,5 $\mu \mathrm{m}$; relación EA/ET 4,6-5,3; estrías 7-8 en $10 \mu \mathrm{m}$; aréolas 20-22 en $10 \mu \mathrm{m}$.

Ecología. - Poco conocida. En las muestras analizadas se la encontró solo como epífita y a una conductividad inferior a $500 \mu \mathrm{s} \mathrm{cm}^{-1}$.

Distribución geográfica. - Para Argentina fue citada como Cymbella minuta var. pseudogracilis (Santiago del Estero y Chaco) y como C. mesiana (Neuquén y Chaco) (Vouilloud, 2003). Nueva cita para Tucumán.

Material estudiado.- ARGENTINA. Prov. Tucumán, Dpto. Lules (Río San Javier), 263' S 6517' O, 880 m snm, 11-12-2014, Nieva 26.415, 26.416, 26.421 (LIL).

\section{Gomphonema laticollum \\ Reichardt, 2001 (Figs. 7C, 7D)}

Descripción.- Valvas notablemente heteropolares, claviformes, débilmente dilatadas en la zona media; ápice de ancho similar a la zona central, con extremos truncadamente redondeados; base angosta con el extremo angostamente redondeado. Área axial angosta, área central irregularmente delimitada, con la estría central más larga que las dos adyacentes. Un estigmoide en el extremo de una de las estrías más largas. Rafe fuertemente lateral. Estrías claramente aereoladas, radiales en la zona media de la valva, levemente paralelas hacia los extremos.

Dimensiones. - Eje apical 40-45,6 $\mu \mathrm{m}$; eje transapical 12,1-13,4 $\mu \mathrm{m} ; 10-11$ estrías en $10 \mu \mathrm{m}$.

Ecología. - La bibliografía aporta poca información. En las muestras se la encontró casi exclusivamente como epífita y toleró altos niveles de conductividad (1146 $\left.\mu \mathrm{s} \mathrm{cm}^{-1}\right)$.

Distribución geográfica.- - Frecuente en regiones tropicales y subtropicales, hallada en una cascada en Uruguay (Mezeltin et al., 2005), aunque también fue encontrada en el Lago Baikal (Kulikovskiy, Kociolek, Solak, Kuznetsova, 2015) y para Argentina, Tucumán (Taboada et al., 2017).

Material estudiado.- ARGENTINA. Prov. Tucumán, Dpto. Lules (Río San Javier), 263' S 65'17' O, 880 m snm, 11-12-2014, Nieva 26.400, 26.415, 26.416, 26.417, 26.418, 26.419, 26.420, 26.421, 26424 (LIL).

Observaciones. - Esta especie no está mencionada para Buenos Aires en el catálogo de Vouilloud (2003). La primera cita para Argentina es de Taboada et al. (2017).

\section{Gomphonema lippertii}

Reichardt y Lange-Bertalot, 1999 (Figs. 7E, 7F)

Descripción. - Valvas lanceoladas a rómbico lanceoladas, claviformes, levemente cimbeloides; ápice angosto, corto con extremo redondeado y base paulatinamente 

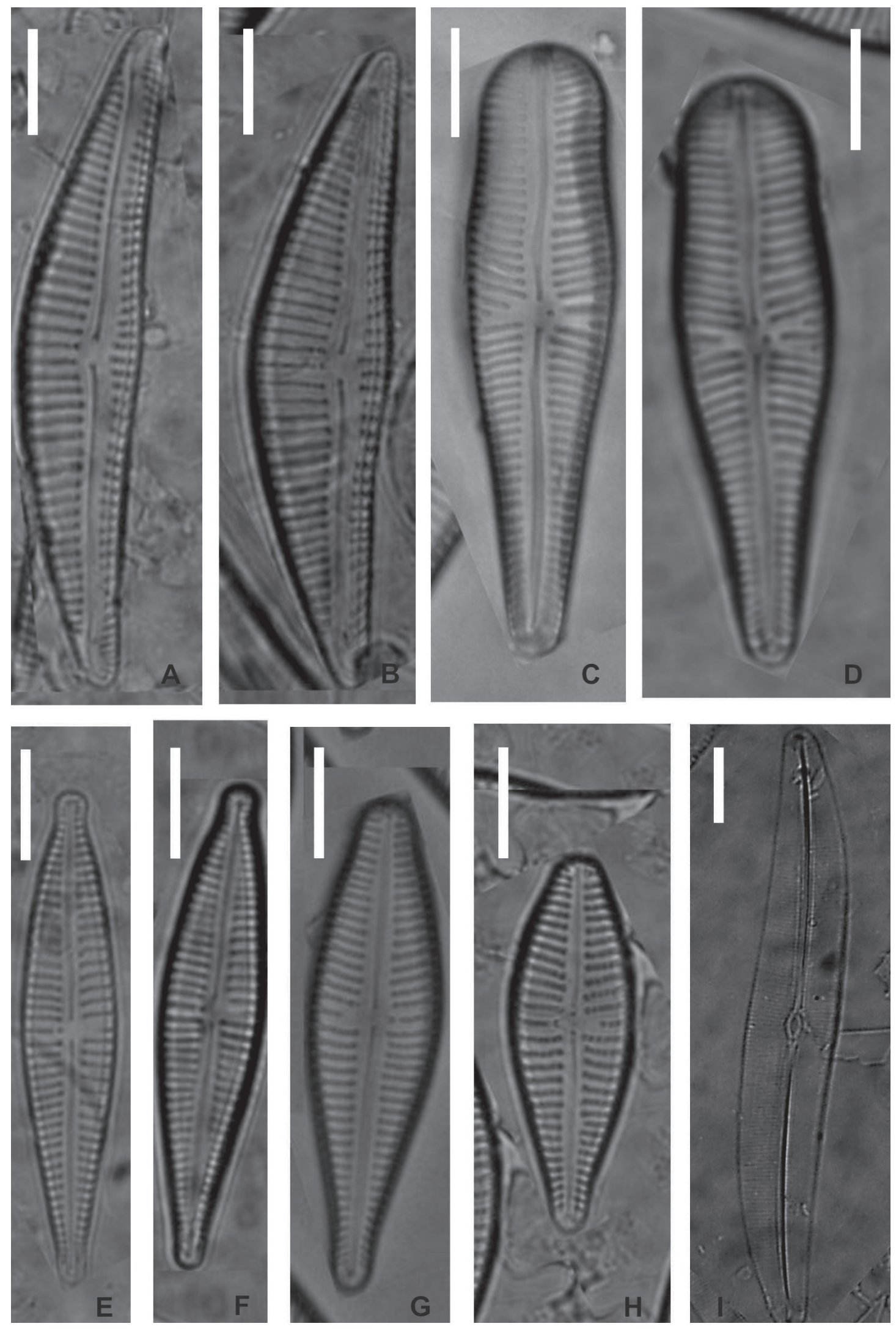

Fig. 7. A-B) Encyonema neomesianum. C-D) Gomphonema laticollum. E-F) Gomphonema lippertii. G-H) Gomphonema mexicanum. I) Gyrosigma sp. Escala $=10 \mu \mathrm{m}$ 
atenuada. Área axial angosta, lineal; área central unilateral delimitada por estrías acortadas; un estigma ubicado cerca del nódulo central, algo separado de la estría media. Rafe lateral, algo curvado a casi recto. Estrías débilmente radiales y en los extremos más densamente dispuestas. Aréolas difíciles del distinguir al MO.

Dimensiones.—Eje apical 42,7-56,4 $\mu \mathrm{m}$; eje transapical 11-12 $\mu \mathrm{m}$; estrías 10-11 en $10 \mu \mathrm{m}$

Ecología.- Reichardt (1999) la menciona para aguas con alto contenido carbonático. Se la encontró en muestras de sitios con conductividad elevada $\left(1146 \mu \mathrm{s} \mathrm{cm}^{-1}\right)$ y se la halló con una alta frecuencia de ocurrencia como epifítica y epilítica.

Distribución geográfica. - mencionada para Europa (Reichardt, 1999). Nueva cita para Argentina.

Material estudiado.- ARGENTINA. Prov. Tucumán, Dpto. Lules (Río San Javier), 2639' S 65'17' O, 880 m snm, 11-12-2014, Nieva 26.400, 26.401, 26.402, 26.403, 26.404, 26405, 26.406, 26.407, 26.408, 26.409, 26.410, 26.411, 26.412, 26.413, 26.414, 26.415, 26.416, 26.417, 26.418, 26.419, 26.420, 26.421, 26.422, 26.423, 26.424 (LIL).

\section{Gomphonema mexicanum}

Grunow, 1880 (Figs. 7G, 7H)

Descripción.- Valvas elípticas, claviformes; ápice diferenciado, levemente rostrado y redondeado; base paulatinamente atenuada. Área axial lineal, ligeramente expandida por el acortamiento de las estrías a cada lado del área central; un estigma alargado, como una hendidura, algo separado de la línea media. Rafe lateral, algo curvado. Estrías paralelas a débilmente radiales. Aréolas claramente distinguibles al MO.

Dimensiones.- Eje apical 23-71 $\mu \mathrm{m}$; eje transapical 9,7-15 $\mu \mathrm{m}$; estrías 8-10 en $10 \mu \mathrm{m}$.

Ecología.- Prefiere aguas cálidas con contenido electrolítico medio a alto y débilmente salinos (Reichardt, 1999). Se la encontró con alta frecuencia de ocurrencia (mayor al $80 \%$ ), en un amplio rango de salinidad, como epífita y epilítica.

Distribución geográfica.- Cosmopolita, reportada para Norte América (Reichardt, 1999) y en Argentina para Tucumán por Taboada et al. (2017).

Material estudiado.- ARGENTINA. Prov. Tucumán, Dpto. Lules (Río San Javier), 263' S 65'17' O, $880 \mathrm{~m} \mathrm{snm}, 11-12-2014$, Nieva 26.400, 26.401, 26.402, 26.403, 26.404, 26405, 26.406, 26.407, 26.408, 26.409, 26.410, 26.411, 26.412, 26.413, 26.414, 26.415, 26.416, 26.417, 26.418, 26.419, 26.420, 26.421, 26.422, 26.423, 26.424 (LIL).

\section{Gyrosigma sp. (Fig. 7I)}

Descripción. - Valvas débilmente sigmoideas, con lados casi paralelos. Extremos algo curvados y ápices trunco-redondeados. Área axial muy angosta; área central pequeña, algo inclinada y longitudinalmente elíptica. Estrías transapicales más evidentes que las longitudinales, débilmente radiales a paralelas. 
Dimensiones. - Eje apical 59-92 $\mu \mathrm{m}$; eje transapical 9,8-13 $\mu \mathrm{m}$; estrías transapicales 21-22 en $10 \mu \mathrm{m}$, estrías longitudinales 20-22 en $10 \mu \mathrm{m}$.

Observaciones. - El material no pudo ser determinado a nivel de especie por no haber podido realizar observaciones con microscopio electrónico de barrido (MEB)

Material estudiado.- ARGENTINA. Prov. Tucumán, Dpto. Lules (Río San Javier), 2639' S 65¹7' O, 880 m snm, 11-12-2014, Nieva 26.400, 26.402, 26.403, 26.404, 26.405, 26.407, 26.415, 26.416, 26.421, 26.423 (LIL).

\section{Halamphora normanii}

(Rabenhorst) Levkov, 2009 (Fig. 8C)

Descripción. - Valvas semilanceoladas, con el margen dorsal arqueado y el ventral recto o ligeramente convexo. Extremos de las valvas proyectados, capitados y ventralmente curvados. Área axial ancha, más notable ventralmente. Área central sobre el lado dorsal de la valva con un semi-estauro delimitado cerca del margen dorsal por unas pocas estrías cortas y distanciadas. Ramas del rafe arqueadas con extremos proximales curvados dorsalmente. Las estrías ventrales son de difícil de ver al MO.

Dimensiones. - Eje apical 31-43,7 $\mu \mathrm{m}$, eje transapical 4-6 $\mu \mathrm{m}$; estrías 18-20 en $10 \mu \mathrm{m}$.

Características ecológicas. - Poco conocidas, en aguas dulces con distinto grado de perturbación (Levkov, 2009). Se la encontró en la mayoría de los casos como epilítica y en muestras con baja salinidad.

Distribución geográfica.- Cosmopolita, ampliamente distribuida en Europa, en Argentina está mencionada para Córdoba como Amphora normanii (Vouilloud, 2003). Es una nueva cita para Tucumán.

Material estudiado.- ARGENTINA. Prov. Tucumán, Dpto. Lules (Río San Javier), 2639' S 65'17' O, 880 m snm, 11-12-2014, Nieva 26.400, 26.404, 26.410, 26.412, 26.413, 26.421 (LIL).

\section{Hantzschia abundans}

Lange-Bertalot, 1993 (Fig. 8A)

Descripción.- Valvas claramente dorsiventrales, con el lado dorsal convexo y el ventral cóncavo en el centro y convexo hacia los extremos. Extremos alargados, rostrados a subcapitados con ápices redondeados a cuneados. Rafe interrumpido en la zona media. Estrías radiales más espaciadas en el centro de la valva y casi paralelas cerca de los ápices. Fíbulas densamente espaciadas con las dos centrales claramente más distantes. Areolas no distinguibles al MO.

Dimensiones.- Eje apical 54-101 $\mu \mathrm{m}$; eje transapical 7-9 $\mu \mathrm{m} ; 18-20$ estrías en $10 \mu \mathrm{m}$, fíbulas 5-8 en $10 \mu \mathrm{m}$. 
Características ecológicas. - Hasta el momento similar a lo reportado para Hantzschia amphioxys y H. subrupestris (Lange-Bertalot, 1993). En las muestras estudiadas, fue hallada como epilítica y epífita en un amplio rango de salinidad.

Distribución geográfica. - Cosmopolita. Para Argentina ha sido citada hasta ahora para la isla Levingston, Antártida, Provincia de Tierra del Fuego (Zidarova, Van de Vijver, Quesada y Hann, 2010). Nueva cita para Tucumán.

Material estudiado.- ARGENTINA. Prov. Tucumán, Dpto. Lules (Río San Javier), 2639' S 6517' O, 880 m snm, 11-12-2014, Nieva 26.400, 26.402, 26.404, 26.405, 26.407, 26.409, 26.415, 26.418, 26.421, 26.424 (LIL).
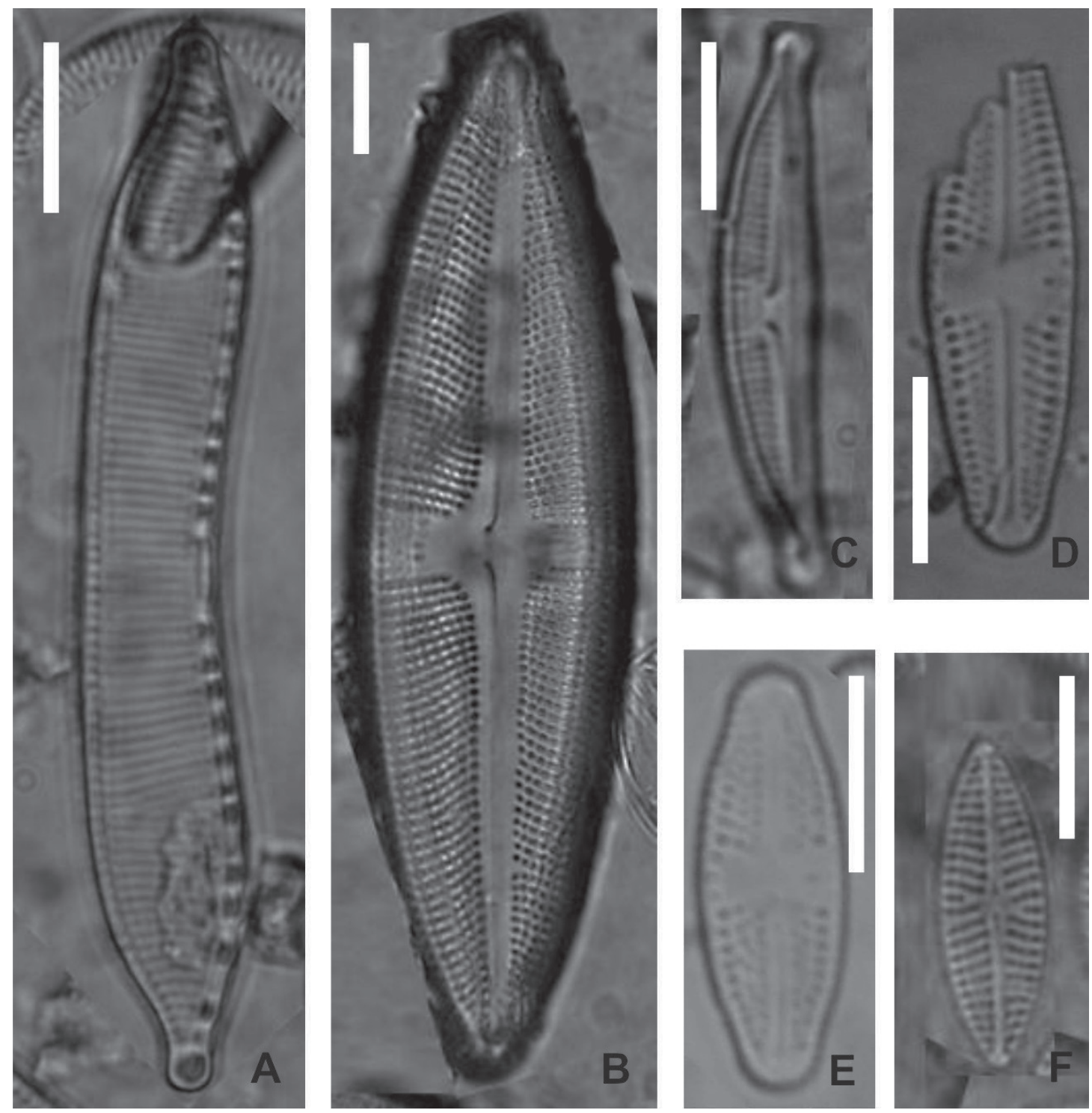

Fig. 8. A) Hantzschia abundans. B) Luticola uruguayensis. C) Halamphora normanii. D-E) Luticola aff. andina. F) Navicula antonii. Escala $=10 \mu \mathrm{m}$ 


\section{Luticola uruguayensis \\ Metzeltin, Lange-Bertalot y García, 2005 (Fig. 8B)}

Descripción.- Valvas lanceoladas, con márgenes levemente ondulados en especímenes grandes a convexo en los más pequeños, con extremos proyectados y ápices obtusamente redondeados. Área axial angosta, lanceolada expandida hacia el área central. Área central variable, levemente asimétrica, limitada por estrías más cortas formadas por 2-3 aréolas. El estigma solitario y alargado transapicalmente. Fisuras proximales del rafe largas, curvadas hacia el lado opuesto del estigma y con extremos en forma de gota. Fisuras distales en forma de gancho. Estrías transapicales radiales compuestas por 8-10 areolas redondeadas a alargadas. Canal marginal muy ancho, casi $1 / 4$ del ancho de la valva.

Dimensiones.- Eje apical 88,7-24,5 $\mu \mathrm{m}$, eje transapical 22,7-23,5 $\mu \mathrm{m} ; 10-11$ estrías en $10 \mu \mathrm{m}$; aréolas 8,8-9,8 en $10 \mu \mathrm{m}$.

Características ecológicas. - Fue encontrada en ríos y arroyos con elevada conductividad. Solo se la encontró como epífítica y en bajos registros de conductividad.

Distribución geográfica.- Fue citada para Misiones, como Navicula mobiliensis var. intermedia Maidana (1983) y para Tucumán (Taboada et al., 2017).

Material estudiado.- ARGENTINA. Prov. Tucumán, Dpto. Lules (Río San Javier), 2639' S 65¹7' O, 880 m snm, 11-12-2014, Nieva 26.415, 26.416 (LIL).

Observaciones: cabe destacar que esta especies fue citada por primera vez para el país por Maidana (1983) como Navicula mobiliensis var. intermedia.

\section{Luticola aff. andina \\ Levkov et al., 2013, (Fig. 8D-E)}

Descripción.- Valvas lineal-lanceoladas con extremos no proyectados y ápices redondeados. Área axial lineal; área central ancha, transversalmente alargada, elíptica a rectangular. Estigma solitario, ubicado entre el centro y el margen. Fisuras proximales del rafe largas, curvadas hacia el lado opuesto del estigma. Fisuras distales largas y en forma de gancho.

Dimensiones.- Eje apical 13,7-24 $\mu \mathrm{m}$, eje transapical 5,6-8 $\mu \mathrm{m}$; estrías 15-18 en $10 \mu \mathrm{m}$.

Características ecológicas. - Fue hallada con alta frecuencia (mayor al $80 \%$ ) en un amplio rango de salinidad como epífita y epilítica.

Observaciones. - Los ejemplares encontrados se asemejan a Luticola andina, pero se diferencian porque las fisuras distales del rafe son largas y curvadas en forma de gancho mientras que en la descripción de la especie son cortas y rectas. Para corroborar la identificación es necesario contar con observaciones con MEB.

Material estudiado.- ARGENTINA. Prov. Tucumán, Dpto. Lules (Río San Javier), 26 $39^{\prime} \mathrm{S} 65^{\circ} 17^{\prime} \mathrm{O}, 880 \mathrm{~m}$ snm, 11-12-2014, Nieva 26.404, 26.407, 26.409, 26.410, 26.411, 26.413, 26.414, 26.415, 26.416, 26.421, 26.424 (LIL). 


\section{Navicula antonii \\ Lange-Bertalot en Rumrich, Lange-Bertalot y Rumrich, 2000}

(Fig. 8F)

Descripción.- Valvas anchamente lanceoladas con extremos cuneados agudos a obtusamente redondeados. Área axial angosta; área central pequeña, irregular. Rafe filiforme. Estrías radiales en la zona media de la valva, paralelas a muy suavemente convergentes en los extremos.

Dimensiones. - Eje apical 12-22 $\mu \mathrm{m}$; eje transapical 5,6-6,8 $\mu \mathrm{m}$; estrías 13-16 en $10 \mu \mathrm{m}$; areolas $28-30$ en $10 \mu \mathrm{m}$.

Características ecológicas.- Común en aguas eutróficas a hipereutróficas, con alto contenido de electrolitos. Indicadora de efectos antrópicos (Hofmann, Werum y Lange-Bertalot, 2011). Se la encontró con una alta frecuencia de ocurrencia (93 \%) y como tolerante a un amplio espectro de salinidad.

Distribución geográfica.- Fue citada para Santa Cruz, Argentina por: Echazú (2012). Nueva cita para Tucumán.

Material estudiado.- ARGENTINA. Prov. Tucumán, Dpto. Lules (Río San Javier), 2639' S 65'17' O, 880 m snm, 11-12-2014, Nieva 26.400, 26.401, 26.402, 26.403, $26.404,26405,26.406,26.407,26.408,26.409,26.410,26.411,26.412,26.414,26.415$, 26.416, 26.417, 26.418, 26.419, 26.420, 26.421, 26.423 (LIL).

\section{Navicula densilineolata}

(Lange-Bertalot) Lange-Bertalot, 1993 (Fig. 9A)

Descripción.- Valvas estrechamente lanceoladas con extremos obtusos y ápices truncado-redondeados. Área axial angosta, lineal; área central pequeña, romboidal, algo asimétrica. Rafe filiforme a débilmente lateral con nódulos centrales ligeramente curvados. Estrías radiales, haciéndose paralelas y luego convergentes en los ápices. Areolas no visibles al MO.

Dimensiones.- Eje apical 26,4-46 $\mu \mathrm{m}$; eje transapical 6,7-7,6 $\mu \mathrm{m}$; estrías 11-12 en $10 \mu \mathrm{m}$.

Características ecológicas. - En aguas duras con contenido electrolítico medio a alto (Hofmann et al., 2011). Se la registró con una frecuencia de ocurrencia del 100 $\%$ como epífita y epilítica.

Distribución geográfica.- Europa (Hofmann et al., 2011). Es una nueva cita para la Argentina.

Material estudiado.- ARGENTINA. Prov. Tucumán, Dpto. Lules (Río San Javier), 2639' S 65'17' O, 880 m snm, 11-12-2014, Nieva 26.400, 26.401, 26.402, 26.403, 26.404, 26405, 26.406, 26.407, 26.408, 26.409, 26.410, 26.411, 26.412, 26.413, 26.414, 26.415, 26.416, 26.417, 26.418, 26.419, 26.420, 26.421, 26.422, 26.423, 26.424 (LIL). 


\section{Navicula rostellata \\ Kützing, 1844 (Fig. 9B)}

Descripción.- Valvas lineales a lineal-lanceoladas, ligeramente convexas, márgenes rectos y con ápices subrostrados. Área axial angosta y recta, área central elíptica y ligeramente asimétrica. $\mathrm{El}$ rafe es recto, con los extremos proximales ligeramente dilatados. Estrías en el centro más distantes, fuertemente radiales y paralelas a convergentes hacia los ápices.

Dimensiones.- Eje apical 40-42 $\mu \mathrm{m}$; eje transapical $9 \mu \mathrm{m}$; estrías $11-12$ en 10 $\mu \mathrm{m}$.

Características ecológicas: cosmopolita, en aguas alcalinas, predominantemente epilítica. Fue muy poco frecuente y solo fue encontrada como epilítica.

Distribución geográfica: Córdoba y Neuquén, Argentina (Vouilloud, 2003). Primera cita para Tucumán.

Material estudiado.- ARGENTINA. Prov. Tucumán, Dpto. Lules (Río San Javier), 2639' S 6517' O, 880 m snm, 11-12-2014, Nieva 26.412 (LIL).

\section{Placoneis neoexigua}

Lange-Bertalot y Miho, 2006 (Fig. 9C)

Descripción.- Valvas elípticas con extremos proyectados, subrostrados y ápices redondeados. Área axial angosta, lineal; área central circular a transversalmente elíptica, sin estigma, delimitada por estrías cortas y largas. Estrías fuertemente radiales.

Dimensiones.- Eje apical 23-24 $\mu \mathrm{m}$; eje transapical 9-10 $\mu \mathrm{m}$; estrías 12 en 10 $\mu \mathrm{m}$.

Características ecológicas: se la encontró en nuestras muestras epilítica, con baja frecuencia de ocurrencia y niveles medios a alto de conductividad $\left(376-886 \mu \mathrm{S} \mathrm{cm}^{-1}\right)$.

Distribución geográfica. - Citada para el lago Ohrid y otros hábitats de agua dulce en Albania (Miho y Lange-Bertalot, 2006). Nueva cita para Argentina.

Material estudiado.- ARGENTINA. Prov. Tucumán, Dpto. Lules (Río San Javier), 263' S 65'17' O, 880 m snm, 11-12-2014, Nieva 26.407 (LIL).

\section{Placoneis symmetrica \\ Lange-Bertalot, 2005 (Fig. 9D)}

Descripción.- Valvas elípticas con extremos proyectados, capitados y ápices redondeados. Área axial angosta, lineal, área central sin estigma, algo difusa, con forma de mariposa y formada por el acortamiento irregular de las estrías. Rafe filiforme con las fisuras proximales rectas. Estrías radiales en toda la valva.

Dimensiones.- Eje apical 26-31 $\mu \mathrm{m}$; eje transapical $10,5-12 \mu \mathrm{m}$; estrías $10-12$ en $10 \mu \mathrm{m}$. 

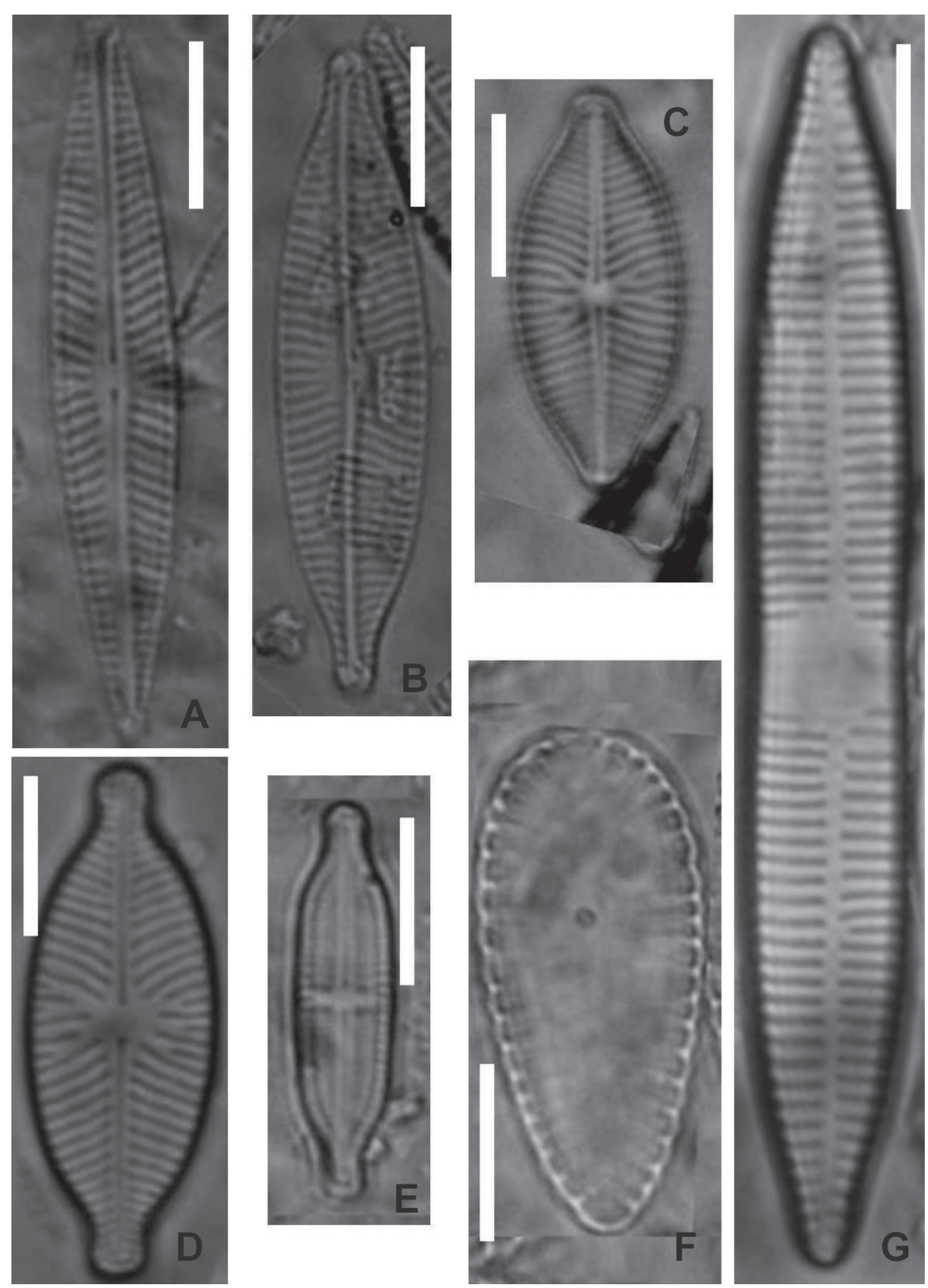

Fig. 9. A) Navicula densilineolata. B) Navicula rostellata. C) Placoneis neoexigua. D) Placoneis symmetrica. E) Stauroneis adamsiana. F) Surirella aff. lacrimula. G) Ulnaria ramesii. Escala $=10 \mu \mathrm{m}$ 
Características ecológicas. - En aguas oligotróficas (Krammer y Lange-Bertalot, 1986). Fue hallada en un amplio espectro de salinidad como epilítica y epífita.

Distribución geográfica.- Citada para Catamarca (Seeligmann y Maidana, 2003) como P. constans var. symmetrica y como Navicula constans var. symmetrica para Río Negro y Neuquén (Vouilloud, 2003). Nueva cita para Tucumán.

Material estudiado.- ARGENTINA. Prov. Tucumán, Dpto. Lules (Río San Javier), 2639' S 65'17' O, 880 m snm, 11-12-2014, Nieva 26.400, 26.403, 26.404, 26.405, 26.406, 26.409, 26.410, 26.411, 26.412, 26.413, 26.415, 26.416, 26.417, 26.418, 26.419, 26.420, 26.421, 26.422, 26.424 (LIL).

\section{Stauroneis adamsiana \\ Metzeltin, Lange-Bertalot y García-Rodríguez, 2005 (Fig. 9E)}

Descripción.- Valvas lineares con márgenes rectos a ligeramente convexos, con extremos proyectados a rostrados y ápices redondeados. Área axial angosta; área central expandida hacia los márgenes de la valva formando una fascia angosta, a menudo con 1-2 estrías fuertemente acortadas. Rafe filiforme, con extremos externos proximales rectos y simples y extremos terminales en forma de gancho, dirigidos hacia el mismo lado de la valva Estrías moderadamente radiadas, subparalelas en los polos.

Dimensiones.- Eje apical 17,5-25,3 $\mu \mathrm{m}$; eje transapical 4-5,7 $\mu \mathrm{m}$; estrías 18-22 en $10 \mu \mathrm{m}$.

Características ecológicas.- No informadas en la bibliografía. Se la encontró, en los sitios estudiados, principalmente como epilítica y con registros de salinidad de hasta $1146 \mu \mathrm{s} \mathrm{cm}^{-1}$.

Distribución geográfica.- Reportada para Uruguay (Metzeltin et al., 2005), Ecuador, Perú (Cocquyt y Van de Vijver, 2007) y Colombia (Montoya, 2012). Nueva cita para Argentina.

Material estudiado.- ARGENTINA. Prov. Tucumán, Dpto. Lules (Río San Javier), 2639' S 6517' O, 880 m snm, 11-12-2014, Nieva 26.400, 26.403, 26.404, 26.407, $26.408,26.409,26.411,26.412,26.414,26.415,26.419$ (LIL).

\section{Surirella aff. lacrimula}

English, 2012 (Fig. 9F)

Descripción.- Valvas netamente heteropolares, ovales a elípticas con un extremo más ancho ampliamente redondeado y el más angosto cuneado-redondeado. Canales alares ausentes; fíbulas prolongadas en costillas que normalmente no alcanzan el centro de la valva. Estrías casi indistinguibles con MO.

Dimensiones.- Eje apical 29-30 $\mu \mathrm{m}$; eje transapical 12,5-13 $\mu \mathrm{m}$; fíbulas 6-7 en $10 \mu \mathrm{m}$. 
Observaciones.- El material estudiado se asemeja en la forma general a la valva de Surirella lacrimula, sin embargo consideramos conveniente contar con mayores registros para precisar la identificación.

Material estudiado.- ARGENTINA. Prov. Tucumán, Dpto. Lules (Río San Javier), 2639' S 6517' O, 880 m snm, 11-12-2014, Nieva 26.413, 26.414 (LIL).

\section{Ulnaria ramesii}

(Héribaud-Joseph) T. Ohtsuka, 2007 (Fig. 9G)

Descripción.- Valvas con extremos rostrados y ápices redondeados. Área axial lineal, angosta; área central elipsoide con estrías fantasma.

Dimensiones.- Eje apical 49-85,2 $\mu \mathrm{m}$; eje transapical 8-10 $\mu \mathrm{m}$; estrías 9-10 en $10 \mu \mathrm{m}$.

Características ecológicas.- Encontrada en el río Ishite (Japón) a una altitud de 700 m snm (Ohtsuka, Nakamura, Nakano y Miyake, 2007).

Distribución geográfica. - Nueva cita para Argentina.

Material estudiado.- ARGENTINA. Prov. Tucumán, Dpto. Lules (Río San Javier), 26 39' S 65'17' O, 880 m snm, 11-12-2014, Nieva 26.400, 26.402, 26.403, 26.404, $26405,26.406,26.407,26.408,26.409,26.410,26.411,26.412,26.413,26.414,26.415$, $26.416,26.417,26.418,26.419,26.420,26.421,26.422,26.423,26.424$ (LIL).

\section{CONCLUSIONES}

Blinn (2001), Potapova y Charles (2003), Licursi, Gómez y Donadelli (2010) y Urrea y Sabater (2009), entre otros, observaron que los cambios de conductividad y la composición iónica influyen en la estructura de la comunidad diatomológica. Hustedt (1957) advirtió que el crecimiento de este grupo algal se veía afectado por la concentración de determinados iones como respuesta a una mayor presión osmótica. Por su parte, Hecky y Kilham (1973) observaron que existe un reemplazo de especies cuando ocurre un incremento de la conductividad.

La zona de estudio incluye el encuentro de un cuerpo de agua dulce con otro de aguas salobres, lo que determina un gradiente de conductividad en el curso de agua principal y el carácter alcalino del agua en el sitio 2. Para el epiliton la especie con mayor abundancia relativa fue Gomphonema parvulum y para el epifiton Cocconeis pediculus.

Estudios realizados por Yehoshua (2002) demostraron que tanto las diatomeas y las clorofitas filamentosas como Cladophora sp. toleraban condiciones de alta intensidad de luz a poca profundidad. Sin embargo, el hallazgo de Cladophora sp. con mayor frecuencia en aguas más profundas sugeriría, que este taxón podría ser menos tolerante a la intensidad de la luz que las diatomeas y más susceptible a las desecaciones y a la abrasión provocada por la turbulencia.

De las 73 especies epilítica registradas para los márgenes y centro en el sitio 1, a partir del ingreso del arroyo Potrerillo, el $70 \%$ fueron también halladas a lo largo 
de toda la transecta y se incorporaron además otras 19 río abajo (Achnanthes coarctata, Caloneis bacillum, C. schumanniana, C. silicula, Denticula valida, Diploneis chilensis, Gomphonema sp. 1, Luticola aff. spauldingiae, Navicula viridula, Navicula sp.,Nitzschia amphibia var. frauenfeldii, $N$. amphibia var. rostrata, N. communis, N. dissipata, N. halloyi, Pinnularia viridis, Placoneis neoexigua, Tryblionella hungarica y T. levidensis), lo que permitió mantener los niveles de riqueza del sitio 1. El número de especies del epifiton también disminuyó al incrementarse la conductividad y, aparentemente, no logró recuperarse como parece haber ocurrido con el epiliton y la riqueza específica de esta comunidad en los sitios 2-5 fue menor que en el sitio 1 .

La mayoría de las especies fueron halladas en sitios con conductividades dentro de su rango de tolerancia, de acuerdo a la literatura consultada (De Wolf, 1982, Van Dam, Mertens, Sinkeldam, 1994, Lowe, 1974, Yves Bey y Ector, 2013, entre otros). Cymbella excisa y Gomphonema parvulum mantuvieron sus niveles de abundancia relativa a lo largo de la transecta. Sin embargo, taxones como Nitzschia amphibia var. amphibia y $N$. fonticola, reportados como tolerantes a altos niveles de mineralización y pH (Yves Be y Ector, 2013) no llegaron a ser dominantes en el sitio 2 y Placoneis symmetrica, que de acuerdo a Hofmann et al., 2011, es frecuente en aguas con contenido electrolítico bajo, se halló en sitios con elevada conductividad.

De las especies aquí descritas, 5 se registraron en todas las muestras (Gomphonema lippertii, G. mexicanum, Navicula antonii, N. densilineolata y Ulnaria ramesi), mientras que Encyonema neomesiana, Gomphonema laticollum Halamphora normanii, Luticola uruguayensis, Navicula rostellata y Placoneis neoexigua se encontraron en 1 o 2 muestras. Estas especies con baja frecuencia de ocurrencia (6-13 \%) fueron exclusivas de una u otra comunidad.

En el análisis de las Bacillariophyceae realizado para el río San Javier se registraron 5 especies que no fueron previamente citadas para Argentina y 6 que son mencionadas por primera vez para Tucumán. El hallazgo de estos nuevos registros pone en evidencia la necesidad de intensificar los estudios taxonómicos, morfológicos y ecológicos de la biodiversidad diatomológica de la cuenca del río Lules.

\section{AGRADECIMIENTOS}

Este trabajo fue financiado por la Secretaría de Ciencia, Arte e innovación Tecnológica (SCAIT) de la UNT con el proyecto: La complejidad de la cuenca del río Lules (Tucumán, Argentina): Estudio de procesos fisicoquímicos y biológicos del sistema.

\section{BIBLIOGRAFÍA}

Bellinger, E. G. y Sigee, D. C. (2010). Introduction to Freshwater Algae. En E. G. Bellinger, D.C. Sige (Eds.), Freshwater Algae: Identification and Use as Bioindicators (pp. 1-40). Chichester, UK: John Wiley \& Sons. 
Blinn, D. W. (2001). Diatom community structure along physicochemical gradients in Saline lakes. Ecology 74: 1246-1263.

Brown, A. D., Pacheco, S., Lomáscolo, T. y Malizia, L. R. (2006). Situación ambiental de los Bosques Andinos Yungueños. En A. D Brown, U. Martínez Ortíz, M. Acerbi, J. Corcuera (Eds.), La situación ambiental Argentina 2005. (pp. 53-61). Buenos Aires: Fundación Vida Silvestre Argentina.

Burns, A. y Ryder, D. S. (2001). Potential for biofilms as biological indicators in Australian riverine systems. Ecological Management $\mathcal{E}$ Restoration 2 (1): 53-64.

Cocquyt, C. y Van de Vijver, B. (2007). La Calera: Diatom composition of a Peruvian hot spring in the Colca canyon. En W. H. Kusber, R. Jahn (Eds.), Proceedings of the 1st Central European Diatom Meeting 2007 (pp. 31-33). Berlin: Botanic Garden and Botanical Museum Berlin-Dahlem.

De Wolf, H. (1982). Method of coding of ecological data from diatoms for computer utilization. Mededelingen Rijks Geologische Dienst 36: 95-110.

Echazú, D. M. (2012). Biodiversidad de diatomeas en humedales del sur de la Provincia de Santa Cruz, Argentina. (Tesis Doctoral), Universidad de Buenos Aires.

Fernández, H. R. y Barber, H. M. (2011). La Cuenca del Río Lules: una aproximación multidisciplinaria a su complejidad. Tucumán, Argentina: EDUNT.

Georgeff, S. M., Ibañez, L., Vides, M. E., Anis, K. B.y Nieva, S. M. (2014). Paleógeno y Neógeno de Tucumán: estratigrafía y paleoambientes sedimentarios. En S. Moyano, M. E. Puchulu, D. S. Fernández, M. E. Vides, S. Nieva, G. Aceñolaza (Eds.), Geología de Tucumán (pp. 106-123). Tucumán: Colegio de Graduados en Ciencias Geológicas de Tucumán.

Hartley, B. (1996). An Atlas of British Diatoms. Bristol, England: P. A. Sims, Biopress Limited.

Hecky, R. E. y Kilham, P. (1973). Diatoms in alkaline, saline lakes: ecology and geochemical implications. Limnology and Oceanography 18 (1): 53-71.

Hofmann, G., Werum, M. y Lange-Bertalot, H. (2011). Diatomeen im Süâwasser - Benthos vom Mitteleuropa. En H. Lange-Bertalot. (Ed.), Bestimmungsflora Kieselalgen für die ökologische Praxis. Über 700 der häufigsten Arten und ihre Ökologie (pp.1- 908). Königstein: Koeltz Botanical Books.

Hustedt, F. (1957). Die Diatomeenflora des Fluss-systems der Weser im Gebiet der Hansestadt Bremen. Abhandlungen der Naturwissenschaftlichen Verein zu Bremen 34 (3): 181-440.

Krammer, K. y Lange-Bertalot, H. (1986). Bacillariophyceae, Band 2/1, Teil: Naviculaceae. Stuttgart, Germany: Gustav Fischer Verlag, Jena.

Krammer, K. y Lange-Bertalot, H. (1988). Bacillariophyceae, 2/2, Teil: Bacillariaceae, Epithemiaceae, Surirellaceae. New York, USA: Gustav Fischer Verlag.

Krammer, K. y Lange-Bertalot, H. (1991). Bacillariophyceae, Band 2/ 3, Teil: Centrales, Fragilariaceae, Eunotiaceae. Stuttgart, Germany: Gustav Fischer Verlag.

Krammer, K. y Lange-Bertalot, H. (2000). Bacillariophyceae, Band 2/ 3, Teil: Centrales, Fragilariaceae, Eunotiaceae (2nd edition). Heidelberg, Alemania: Spektrum Akademischer Verlag. 
Krammer, K. y Lange-Bertalot, H. (2004). Bacillariophyceae. Band 2/4, Teil: Achnanthaceae Kritische Ergänzungen zu Achnanthes s. 1., Navicula s. str., Gomphonema. Stuttgart, Germany: Gustav Fischer Verlag.

Kulikovskiy, M., Kociolek, J. P, Solak, C. y Kuznetsova, N. I. (2015). The diatom genus Gomphonema Ehrenberg in Lake Baikal. II. Revision of taxa from Gomphonema acuminatum and Gomphonema truncatum-capitatum complexes. Phytotaxa 233 (3): 251-272.

Lange-Bertalot, H. (1993). 85 New Taxa and much more than 100 taxonomic clarifications supplementary to Süsswasserflora von Mittlereuropa Vol 2/1-4. Stuttgart: J Cramer.

Lange-Bertalot, H. (1999). Neue Kombinationen von Taxa aus Achnanthes Bory (sensu lato). Iconographia Diatomologica 6: 276-289.

Levkov, Z. (2009). Diatoms of Europa 5. Amphora sensu lato. Germany: A.R.G. Gantner Verlag K. G.

Levkov, Z., Metzeltin, D. y Pavlov, A. (2013). Diatoms of Europe, Diatoms of the European Inland waters and comparable habitats. Luticola and Luticolopsis. Germany: A.R.G. Gantner Verlag,

Licursi, M. y Gómez, N. (2003). Aplicación de índices bióticos en la evaluación de la calidad del agua en sistemas lóticos de la llanura Pampeana argentina a partir del empleo de diatomeas. Biología Acuática 21: 31-49.

Licursi, M., Gómez, N. y Donadelli, J. (2010). Ecological optima and tolerances of coastal bethic diatoms in the freshwater-mixohaline zone of the Río de la Plata estuary. Marine Ecology Progress Series 418: 105-117.

Lowe, R. L. (1974). Environmental requirements and pollution tolerance of freshwater diatoms. Ohio: National Environmental Research Center.

Luchini, L. y Verona, C. A. (1972). Catálogo de las diatomeas argentinas. I. Diatomeas de aguas continentales (incluido el Sector Antártico). Comisión de Investigaciones Científicas de la Provincia de Buenos Aires. Monografía No 2, La Plata.

Maidana, N. I. (1983). Estudio taxonómico de las Diatomeas de Misiones (Argentina): II. Lilloa 36 (1):151-158.

Malizia, L. R., Blendinger, P. G., Álvarez, M. E., Rivera, L. O., Politi, N. y Nicolossi, G. (2005). Bird communities in Andean Premontane forests of Northwestern Argentina. Ornitología Neotropical 16: 231-251.

Metzeltin, D. y Lange-Bertalot, H. (1998). Tropical diatoms of South America I: About 700 predominantly rarely known or new taxa representative of the neotropical flora. Iconographia Diatomologica 5: 1-695.

Metzeltin, D. y Lange-Bertalot, H. (2007). Tropical diatoms of South America II: Special remarks on biogeographic disjunction. Iconographia Diatomologica 18: 1-877.

Metzeltin, D., Lange-Bertalot, H. y García-Rodríguez, F. (2005). Diatoms of Uruguay; taxonomy-biogeography-diversity. Iconographia Diatomologica 15: 1-736.

Miho, A. y Lange-Bertalot, H. (2006). Diversity of the genus Placoneis in Lake Ohrid and other freshwater habitats in Albania. En A. Witkowski (Ed.), Eighteenth International Diatom Symposium (pp 301-313). Miedzyzdroje, Poland. 
Minetti, J. L. y Vargas, W. M. (1997). Trends and Jumps in the annual precipitation in South America, south of the 15²». Atmósfera 11: 200-221.

Montoya-Moreno, Y. (2012). Diatomeas (Bacillariophyta) perifíticas del Complejo cenagoso de Ayapel, Colombia. I. Caldasia 34 (2): 457-474.

Ohtsuka, T., Nakamura, Y., Nakano, S. y Miyake, Y. (2007). Diatoms from Ishite Stream, near the Komenono forest Research Center of Ehime University, Japan. Diatom Research 23: 29-48.

Patrick, R. (1961). Diatoms (Bacillariophyceae) from the alimentary tract of Phoenicoparrus jamesi (Sclater). Postilla 49: 43-55.

Patrick, R. y Reimer, C. W. (1966). The diatoms of the United States (exclusive of Alaska and Hawaii). Vol. 1. Philadelphia: Acad. Nat. Sci.

Patrick, R. y Reimer, C. W. (1975). The diatoms of the United States (exclusive of Alaska and Hawaii). Vol. 2. Philadelphia: Academy of Natural Sciences of Philadelphia.

Pizarro, H. E. y Alemanni, M. E. (2005). Variables físico-químicas del agua y su influencia en la biomasa del perifiton en un tramo inferior del Río Luján (Provincia de Buenos Aires). Ecología Austral 15 (1): 73-88.

Potapova, M. y Charles, D. F. (2003). Distribution of benthic diatoms in US rivers in relation to conductivity and ionic composition. Freshwater Biology 48 (8): 1311-1328.

Reichardt, E. (1999). Zur Revision der Gattung Gomphonema. Die Arten um G. affine/insigne, $G$. angustatum/micropus, $G$. acuminatum y sowie gomphonemoide Diatomeen aus dem Oberoligozán in Böhmena. En H. Lange-Bertalot. (Ed.), Iconographia Diatomologica. Annotated Diatom Micrographs. Taxonomy. Vol. 8 (pp. 5-203) Königstein: Koeltz Scientific Boks, ARG Gantner.

Reichardt, E. (2001). Revision der Arten um Gomphonema truncatum und G. capitatum. En: R. Jahn, J. P. Kociolek, A. Witkowski, P. Compère (Eds.). Studies on diatoms dedicated to Prof. Dr. Horst Lange-Bertalot on the occasion of his 65th birthday. (pp. 187-224). Ruggell: A.R.G. Gantnter Verlag K.G.

Rumrich, U., Lange-Bertalot, H. y Rumrich, M. (2000). Diatomeen der Anden von Venezuela bis Patagonien/Tierra del Fuego. En H. Lange-Bertalot (Ed.), Iconographia Diatomologica 9 (pp. 1-672). Ruggell: A.R.G. Gantner Verlag K. G.

Seeligmann C. T. y Maidana, N. I. (2003). Diatomeas de la provincia de Catamarca (Argentina). Boletín de la Sociedad Argentina de Botánica 38: 39-50.

Simler, R. (2009). Diagrammes. Avignon, Laboratoire d'Hydrogéologie d'Avignon. http://www.lha.univ-avignon.fr/LHA-Logiciels.htm.

Stevenson, R. J., Peterson, C. G., Kirschtel, D. B., King, C. C. y Tuchman, N. C. (1991). Density dependent growth, ecological strategies, and effects of nutrients and shading on benthic diatom succession in streams. Fournal of Phycology 27 (1): 59-69.

Stevenson, R. J., Yangdong, P. y Van Dam, H. (2010). Assessing environmental conditions in rivers and streams with diatom. En J. P. Smol, E. F. Stoermer. (Eds.), The Diatoms: Applications for the Environmental and Earth Sciences 2nd Edition (pp. 57-85). Cambridge: Cambridge University Press. 
Taboada, M. A., Martínez De Marco, S., Tracanna, B. y Bustos, S. (2017). Nuevos registros de Bacillariophyceae en ecosistemas lóticos del Noroeste de Argentina I. Lilloa 54 (2): 240-256.

Tell, G. y Mazzoni, H. (1995). Perifiton: colonización, sucesión, productividad. En E. C. Lopreto, G. Tell, (Eds.), Ecosistemas de aguas continentales, metodologías para su estudio. Vol. 1 (pp. 161-168). Buenos Aires: Ediciones Sur.

Urrea, G. y Sabater S. (2009). Epilithic diatom assemblages and their relations to environmental characteristics in an agrigultural watershed (Guadiana River), SW Spain). Ecological Indicators 9: 693-703.

Van Dam, H., Mertens, A. y Sinkeldam, J. (1994). A coded checklist and ecological indicator values of freshwater diatoms from the Netherlands. Netherlands $\mathcal{F o}_{0}$ urnal of Aquatic Ecology 28: 117-133.

Villafañe, V. E. y Raid, F. M. (1995). Métodos de microscopía para la cuantificación del fitoplancton. En K. Alveal, M. E. Ferrario, E. C. Oliveira y E. Sar (Eds.), Manual de Métodos Ficológicos (pp. 169-185). Universidad de Concepción, Chile.

Vouilloud, A. (2003). Catálogo de diatomeas continentales y marinas de Argentina. Versión 1.0. En soporte magnético. Asociación Argentina de Ficología, La Plata.

Wetzel, R. G. (1983). Attached algal-substrata interactions: fact or myth, and when and how?. En: R. G. Wetzel (Ed.), Periphyton of Freshwater Ecosystems. Vol.17 (pp 207-215). Developments in Hydrobiology. Dordrecht: Springer.

Yehoshua, Y. (2002). The lake Kinneret epilithon: ecophysiology and function in the ecosystem. Ramat- Gam, Israel: Bar-Ilan University.

Yves Bey, M. y Ector, L. (2013). Atlas des diatomées des cours d'eau de la région Rhône-Alpes. Caluire, Francia: Bonn'Impression.

Zidarova, R., Van de Vijver, B., Quesada, A. y Hann, M. (2010). Revision of the genus Hantzschia (Bacillariophyceae) on Linvingston Island (South Islands, Southern Atlantic Ocean). Plant Ecology and Evolution 143 (3): 318-333. 